\title{
Novel Localized Waves and their Interaction Solutions for a Dimensionally Reduced (2+1)- dimensional Boussinesq Equation from $\mathrm{N}$-soliton Solutions
}

\section{Dipankar Kumar ( $\sim$ dks.bsmrstu@gmail.com )}

Bangabandhu Sheikh Mujibur Rahman Science and Technology University https://orcid.org/00000003-2949-166X

\section{Md. Nuruzzaman}

Bangabandhu Sheikh Mujibur Rahman Science and Technology University

Gour Chandra Paul

University of Rajshahi

\section{Ashabul Hoque}

Bangabandhu Sheikh Mujibur Rahman Science and Technology University

\section{Research Article}

Keywords: Boussinesq equation, Hirota bilinear method, Long-wave limit approach, N-solitons, Localized waves, Interaction solutions.

Posted Date: July 14th, 2021

DOI: https://doi.org/10.21203/rs.3.rs-666725/v1

License: (c) (i) This work is licensed under a Creative Commons Attribution 4.0 International License.

Read Full License

Version of Record: A version of this preprint was published at Nonlinear Dynamics on January 31st, 2022. See the published version at https://doi.org/10.1007/s11071-021-07077-9. 
${ }^{*}$ Corresponding author Email addresses: dks.bsmrstu@gmail.com (D. Kumar); nafizmath95@gmail.com (M. Nurruzzaman); gcpaul@ru.ac.bd (G. C. Paul); ashabulh@yahoo.com (A. Hoque) 


\section{Introduction}

The research of localized waves is one of the foremost topics in the areas of nonlinear science and mathematical physics. In nonlinear science, solitons, lumps, breathers, and rogue waves are localized waves, which are important objects in nonlinear physical systems [1]. Due to its importance in nonlinear science, researchers have paid deep attention to the exploration of these localized waves to a family of nonlinear evolution equations (NLEEs). Generally, solitary waves are localized waves that propagate at a constant speed without changing their shape [2]. Such a wave shape preserves its identity after pair-wise collisions, caused by a cancellation of nonlinear and dispersive effects in any nonlinear models [2]. With numerical simulations, Hirota [3] validated the preservation of soliton identities after pair-wise collisions. But the detailed analysis of the numerical results exposed the existence of some ripples after a collision meaning that the original identity is not completely recovered [4]. Therefore, it is directive to explore exact solutions of NLEEs admitting soliton solutions for proper scrutiny of collisions.

It is worth mentioning that John Scott Russell, in 1834, first observed a solitary wave travelling along a Scottish canal, and thereafter in 1965, Zabusky and Kruskal introduced the term "soliton" $[5,6]$. However, on the other hand, lumps are rationally decaying solutions that are localized in all spatial directions, while breathers are partially localized breathing waves with a periodic structure in one certain direction [7]. A rogue wave is a special kind of rational solution that is localized both in spatial and temporal directions $[8,9]$. The mechanism of rogue/freak waves can be regarded as the high amplitude waves generated by the interaction of solitons and breathers $[1,10]$. The concept of rogue/ freak waves in the ocean was first proposed by Draper in 1965 [1]. The nonlinear localized waves mentioned above (solitons, lumps, breathers, and rogue waves) appear in several fields, such as oceanography, fluids, plasma physics, mathematical physics, optical fibers, nonlinear optics, cold atoms, Bose- 
57 Einstein condensates, and so on [11-14]. These localized waves also illustrate various significant instances in nature via some of their related nonlinear models. Besides, interactions among different localized waves are also an interesting topic in nonlinear science [15]. Regarding the interest, researchers have focused on the exploration of some novel interaction solutions among four types of nonlinear localized waves to the NLEEs.

Nowadays, analytic solutions have a great contribution in the diverse field of nonlinear science and physics for interpreting the hidden mechanism via some relevant NLEEs. In order to interpret such underlying mechanism to their nonlinear models, several effective methods have been developed and performed sophistically due to the accessibility of symbolic computation software that make it possible to perform the intricate and tedious calculations. Some examples of the effective analytic methods are the inverse scattering transform [16], the Backlund transformation [17], the Darboux transformation [18], the Kudryashov method [19], the modified Kudryshov method [20, 21], the generalized Kudryashov method [22], the Jacobi elliptic function expansion method [23], the sine-Gordon expansion method [24], the extended sinh-Gordon expansion method [25-27], the $\left(G^{\prime} / G, 1 / G\right)$-expansion method [28], the auxiliary equation method [29], the new auxiliary equation method [22, 30], the variable separation method [31], the Riemann-Hilbert space approach [7, 32], the Painlevé analysis [33], the Consistent Riccati expansion method [34], and the HBM [3, 9, 35-38].

Among the aforementioned methods, researchers have proved that the HBM is widely popular due to its simplicity and directness, which is particularly useful to derive $N$-soliton solutions to any nonlinear integrable models. Recently, with the use of the long-wave limit approach in coordination with constraint parameters, the localized waves including solitons, lumps, breathers, rogue waves, and their interaction solutions from $N$-soliton solutions have been found through a variety of nonlinear models, which are mostly published in 2018-2020 $[1,10,39-48]$. Among the cited refs. [1, 10, 39-48], no significant studies have been found for 
analyzing the wave propagation dynamics of the localized waves and their interaction structures in shallow water through some relevant models. Although, in the past, Wazwaz [49] mentioned that the study of wave propagation of long waves in shallow water has become a dynamic research field in nonlinear science. As in ref. Jawad et al. [50], the dynamics of wave propagation in shallow water are seen in lakes, rivers, ocean beaches and sea, and described by the $\mathrm{BqE}$, which leads to

$$
u_{t t}-u_{x x}-\beta\left(u^{2}\right)_{x x}-\gamma u_{x x x x}=0 .
$$

The BqE was first introduced by Joseph Boussinesq in the 1870s for modeling the propagation of shallow water waves in multiple directions [51]. In this regard, the $\mathrm{BqE}$ contributes to a dominant role in explaining different physical aspects in the field of ocean engineering, fluid dynamics, and plasma physics dealing with a variety of wave phenomena. In ocean and coastal engineering, oceanographers and coastal engineers use the BqEs for simulating surface water waves in shallow seas and harbors, dune, ocean basin-scale tsunami propagation, wave over-topping, inundation, and near shore wave process modeling in which nonlinearities and dispersion are seen to be taken into consideration [52-57]. It is of interest to note here that the physical behaviors of any nonlinear models can be explored by analyzing their localized waves and interaction solutions. The Boussinesq $(\mathrm{Bq})$ model is one of the ideal models for simulating wave propagation on ocean surfaces. To study the wave propagation dynamics on the ocean surfaces, we will construct the nonlinear localized waves and their interaction solutions to a new integrable $\mathrm{Bq}$ model. For this purpose, first, we consider the new integrable (3+1)-dimensional BqE [52], which leads to

$$
u_{t t}-u_{x x}-\beta\left(u^{2}\right)_{x x}-\gamma u_{x x x x}+\frac{\alpha^{2}}{4} u_{y y}+\alpha u_{y t}+\delta u_{x z}=0,
$$

where $\alpha, \beta, \gamma, \delta$ are arbitrary constants, and $u$ is an unknown function that depends on the spatial coordinates $(x, y, z)$, and temporal coordinate $t$. Eq. (2) explains the propagation of gravity waves on the water surface [52]. Wazwaz and Kaur [52] proposed the extended form 
of $\mathrm{BqE}$ specified by Eq. (2) to testify the integrability conditions via the Painlevé test. The authors constructed real and complex multiple soliton solutions via the simplified Hirota's method, and some novel solutions via the $\exp (-\varphi(\eta))$-expansion method.

It is noteworthy to mention here that Eq. (2) can be converted into the standard fourthorder BqE given by Eq. (1) if we set each of $\alpha$ and $\delta$ equal to zero. On the other hand, if we put $\delta=0$ in Eq. (2), then it reduces to the $(2+1)$-dimensional $\mathrm{BqE}$, which is consistent with that of Wazwaz and Kaur [52]. It is convenient to reduce Eq. (2) from (3+1)-dimensions to (2+1)-dimensions by setting $z=0, z=x$, and $z=y$. If we set $z=0$ in Eq. (2), it is converted to the new integrable (2+1)-dimensional $\mathrm{BqE}$, which can be found in Wazwaz and Kaur [52]. However, if we set $z=x$ and $\delta=1$ to Eq. (2), it is converted to (2+1)-dimensional BqE, but the second-order linear dispersion term $\left(u_{x x}\right)$ of the equation will disappear. Therefore, this paper is devoted to the study of dimensionally reduced (2+1)-dimensional $\mathrm{BqE}$ to construct the localized waves and their interaction solutions by setting $z=y$ in Eq. (2), which leads to

$$
u_{t t}-u_{x x}-\beta\left(u^{2}\right)_{x x}-\gamma u_{x x x x}+\frac{\alpha^{2}}{4} u_{y y}+\alpha u_{y t}+\delta u_{x y}=0
$$

It is remarkable to mention here that Eq. (3) must follow the integrability conditions. In the past, the dimensionally reduced form of the generalized Kadomtsev-Petviashvili (gKP) and Boiti-Leon-Manna-Pempinelli (BLMP) equations were solved by Liu et al. [48] and Wu et al. [10], respectively, where both the group of investigators constructed the localized waves and their interaction solutions from $N$-soliton solutions via the HBM in coordination with longwave limit approach presented in Ablowitz and Satsuma [58]. For constructing the localized waves and their interaction wave solutions to the gKP and BLMP equations, Liu et al. [48] reduced the (3+1)-dimensional gKP equation dimensionally after substituting $z=x$, and $\mathrm{Wu}$ et al. [10] reduced the (3+1)-dimensional BLMP equation dimensionally after substituting $z=$ 0 and $z=x$. To the best of the authors' knowledge, the localized waves and their novel interaction solutions have never been reported, so far, to the dimensionally reduced $(2+1)$ - 
dimensional $\mathrm{BqE}$ given by Eq. (3). Therefore, the key aim of the study is to construct nonlinear localized waves and their variety of interaction solutions among the localized waves to the dimensionally reduced (2+1)-dimensional Hirota bilinear form $\mathrm{BqE}$ from $N$-solitons solutions via the long-wave limit approach in conjunction with some constraint parameters. It is to be pointed out here that the long-wave limit approach in coordination with some constraint parameters is relatively different over the direct search positive quadratic function, hyperbolic function, trigonometric function, and their combinations, and the homoclinic test function approaches, which can be found in refs. [35-38].

The organization of the paper is as follows. Sec. $\$ 2$ deals with the $N$-soliton solutions of the new integrable $(3+1)$-dimensional $\mathrm{BqE}$ and its dimensionally reduced $(2+1)$-dimensional $\mathrm{BqE}$ via the HBM. Sec. $\$ 3$ presents nonlinear localized waves and their novel interaction solutions of the reduced (2+1)-dimensional $\mathrm{BqE}$ from $N$-soliton solutions taking the long-wave limit approach in coordination with some constraint parameters. Finally, the discussion of the obtained results and conclusion are placed in Sec.§4.

\section{2. $N$-soliton solutions to the novel integrable (3+1)-dimensional $\mathrm{BqE}$ and its dimensionally reduced from}

We use the following dependent variable transformation [52]:

$u=\frac{6 \gamma}{\beta}(\ln f)_{x x}$

where $f(x, y, z, t)$ is an unknown function of spatio-temporal coordinates $x, y, z$, and $t$.

Eq. (2) under the transformation given by Eq. (4) is then transformed into the Hirota bilinear equation (HBE) as

$\left(D_{t}^{2}-D_{x}^{2}-\gamma D_{x}^{4}+\frac{1}{4} \alpha^{2} D_{y}^{2}+\alpha D_{y} D_{t}+\delta D_{x} D_{z}\right)(f . f)=0$.

In Eq. (5), $D_{x}, D_{y}, D_{z}$, and $D_{t}$ stand for Hirota's bilinear differential operators (HBDOs) defined by (see Kumar et al. [38])

$D_{x}^{\alpha} D_{y}^{\beta} D_{z}^{\gamma} D_{t}^{\eta}(f \cdot g)=\left.\left(\frac{\partial}{\partial x}-\frac{\partial}{\partial x^{\prime}}\right)^{\alpha}\left(\frac{\partial}{\partial y}-\frac{\partial}{\partial y^{\prime}}\right)^{\beta}\left(\frac{\partial}{\partial z}-\frac{\partial}{\partial z^{\prime}}\right)^{\gamma}\left(\frac{\partial}{\partial t}-\frac{\partial}{\partial t^{\prime}}\right)^{\eta} f(x, y, z, t) g\left(x^{\prime}, y^{\prime}, z^{\prime}, t^{\prime}\right)\right|_{x=x^{\prime}, y=y^{\prime}, z=z^{\prime} t=t^{\prime}}$, 
where $\alpha, \beta, \gamma$, and $\eta$ are non-negative integers.

By virtue of the definition of HBDO and its properties, Eq. (5) is turned into the following form:

$f_{t t} f-f_{t}^{2}-f_{x x} f+f_{x}^{2}-\gamma\left(f_{x x x x} f-4 f_{x x x} f_{x}+3 f_{x x}^{2}\right)+\frac{1}{4} \alpha^{2}\left(f_{y y} f-f_{y}^{2}\right)+\alpha\left(f f_{y t}-\right.$

$\left.f_{y} f_{t}\right)+\delta\left(f_{x z} f-f_{x} f_{z}\right)=0$

Based on the simplified HBM and the transformation given by Eq. (4), $N$-soliton solutions are obtained from

$f_{i}=1+\sum_{i=1}^{N} e^{\theta_{i}}+\sum_{i<j}^{N} a_{i j} e^{\left(\theta_{i}+\theta_{j}\right)}+\sum_{i<j<k}^{N} a_{i j} a_{i k} a_{j k} e^{\left(\theta_{i}+\theta_{j}+\theta_{k}\right)}+\cdots+\left(\prod_{i<j} a_{i j}\right)\left(e^{\Sigma_{i=1}^{N} \theta_{i}}\right)$,

where $\theta_{i}$ and $a_{i j}$ stand for denoting the dispersion variables and generalized phase shifts, respectively, and are given by

$\theta_{i}=a_{i} x+b_{i} y+c_{i} z+\left(-\frac{1}{2} \alpha b_{i}-\sqrt{\gamma a_{i}^{4}-\delta a_{i} c_{i}+a_{i}^{2}}\right) t+\omega_{i}, i=1,2, \ldots, N$, and $a_{i j}=\frac{4 \gamma a_{i}^{3} a_{j}-6 \gamma a_{i}^{2} a_{j}^{2}+4 \gamma a_{i} a_{j}^{3}-\delta a_{i} c_{j}-\delta a_{j} c_{i}-2 \sqrt{a_{i}\left(\gamma a_{i}^{3}-\delta c_{i}+a_{i}\right)} \sqrt{a_{j}\left(\gamma a_{j}^{3}-\delta c_{j}+a_{j}\right)}+2 a_{i} a_{j}}{4 \gamma a_{i}^{3} a_{j}+6 \gamma a_{i}^{2} a_{j}^{2}+4 \gamma a_{i} a_{j}^{3}-\delta a_{i} c_{j}-\delta a_{j} c_{i}-2 \sqrt{a_{i}\left(\gamma a_{i}^{3}-\delta c_{i}+a_{i}\right)} \sqrt{a_{j}\left(\gamma a_{j}^{3}-\delta c_{j}+a_{j}\right)}+2 a_{i} a_{j}}, i, j=1,2, \ldots, N$,

where $a_{i}, b_{i}, c_{i}$, and $\omega_{i}$ are arbitrary constants associated with the amplitude and phase of the $i$ th-soliton. It is pertinent to mention that Wazwaz and Kaur [52] have recently constructed the multiple solitons for Eq. (1) by using the above expression. So, we will not repeat its physical importance.

Without loss of generality, $z=y$ can be set in Eq. (5). Then, Eq. (5) is converted to the following $\mathrm{HBE}$ :

$$
\left(D_{t}^{2}-D_{x}^{2}-\gamma D_{x}^{4}+\frac{\alpha^{2}}{4} D_{y}^{2}+\alpha D_{y} D_{t}+\delta D_{x} D_{y}\right)(f . f)=0
$$

Also, the functions for obtaining $N$-soliton solutions of Eq. (10), the dispersion variables $\left(\theta_{i}\right)$, and the generalized phase shifts $\left(a_{i j}\right)$ are changed, respectively, into $f_{i}=1+\sum_{i=1}^{N} e^{\theta_{i}}+\sum_{i<j}^{N} a_{i j} e^{\left(\theta_{i}+\theta_{j}\right)}+\sum_{i<j<k}^{N} a_{i j} a_{i k} a_{j k} e^{\left(\theta_{i}+\theta_{j}+\theta_{k}\right)}+\cdots+\left(\prod_{i<j} a_{i j}\right)\left(e^{\Sigma_{i=1}^{N} \theta_{i}}\right)$, 
181 where $\theta_{i}=a_{i} x+b_{i} y-\left(\frac{1}{2} \alpha b_{i}+\sqrt{\gamma a_{i}^{4}-\delta a_{i} b_{i}+a_{i}^{2}}\right) t+\omega_{i}, i=1,2, \ldots, N$,

and

$a_{i j}=\frac{4 \gamma a_{i}^{3} a_{j}-6 \gamma a_{i}^{2} a_{j}^{2}+4 \gamma a_{i} a_{j}^{3}-\delta a_{i} b_{j}-\delta a_{j} b_{i}-2 \sqrt{a_{i}\left(\gamma a_{i}^{3}-\delta b_{i}+a_{i}\right)} \sqrt{a_{j}\left(\gamma a_{j}^{3}-\delta b_{j}+a_{j}\right)}+2 a_{i} a_{j}}{4 \gamma a_{i}^{3} a_{j}+6 \gamma a_{i}^{2} a_{j}^{2}+4 \gamma a_{i} a_{j}^{3}-\delta a_{i} b_{j}-\delta a_{j} b_{i}-2 \sqrt{a_{i}\left(\gamma a_{i}^{3}-\delta b_{i}+a_{i}\right)} \sqrt{a_{j}\left(\gamma a_{j}^{3}-\delta b_{j}+a_{j}\right)}+2 a_{i} a_{j}}, i, j=1,2, \ldots, N$.

Using Eq. (11) with the aid of Eqs. (12) and (13) in Eq. (4), one can obtain the following $N$ soliton solutions of Eq. (2):

$u=\frac{6 \gamma}{\beta}\left(\ln f_{i}\right)_{x x}, i=1,2, \ldots, N$,

where $f_{i}(x, y, t)$ is a function involving spatial coordinates $x, y$, and temporal coordinate $t$.

Taking $N=1, N=2, N=3$, and $N=4$ to Eq. (14) along with Eqs. (11)-(13), one can acquire one-stripe, two-stripe, three-stripe, and four-stripe bright solitons, respectively, of Eq.

(3). These bright solitons are displayed in Figs. 1(a)-(d) under the suitable selection of the constraint parameters as $a_{1}=1, a_{2}=-1, a_{3}=2, a_{4}=-2, b_{1}=1, b_{2}=-1, b_{3}=2, b_{4}=$ $-2, \omega_{1}=0, \omega_{2}=0, \omega_{3}=0, \omega_{4}=0, \alpha=1, \beta=1, \gamma=1, \delta=1$, and $y=0$. Furthermore,

Figs. 1(e)-(h) exhibit the corresponding density views of Figs. 1(a)-(d). It is realized visually height, width, and speed before $t<0$ and after $t>0$. During interactions, the solitons change only their phases. Therefore, the interactions among the $N$-solitons are elastic. Now, if we set $\beta=-1$ instead of $\beta=1$ in the $N$-soliton solutions given by Eq. (14), the bright type $N$ solitons are changed into the dark types in the $(x, t)$-plane. It is to be mentioned here that the interactions of the dark solitons maintain the similar behaviors as that of the bright solitons.

\section{Localized waves and their interaction solutions among $\boldsymbol{N}$-soliton solutions}

In this section, we will discuss localized waves and their interaction solutions in the case of two-stripe, three-stripe, and four-stripe solitons. 


\subsection{Interaction solutions between two-stripe solitons}

Here, we discuss two cross-stripe solitons, parallel-stripe solitons, $x$-periodic breather, $y$ periodic breather, lump, and rogue wave solutions of Eq. (3). By taking $N=2$ in Eq. (11), one can derive a function for two-soliton solutions given by

$f_{2}=1+e^{\theta_{1}}+e^{\theta_{2}}+a_{12} e^{\theta_{1}+\theta_{2}}$

where $\theta_{i}$ and $a_{i j}$ are given by Eqs. (12) and (13), respectively. Substitution of Eq. (15) into Eq. (14), yields the two-soliton solutions of Eq. (3) as

$u=\frac{6 \gamma}{\beta}\left(\ln f_{2}\right)_{x x}$

\section{Case I: Two cross-stripe and two parallel-stripe solitons}

In order to determine the intersection solutions between two stripe solitons of Eq. (3), the constraint parameters $a_{i}, b_{i}$, and $\omega_{i}$ need to satisfy the following conditions for Eq. (16) along with Eq. (15):

$a_{i}=p_{i}, b_{i}=q_{i},(i=1,2)$,

where $p_{i}$ and $q_{i}$ are real constants, $p_{i} \neq 0$, and $q_{1} \neq q_{2}$.

For cross-stripe solitons, the cross-product of $p_{1}, p_{2}, q_{1}$, and $q_{2}$ follows $\left(p_{1} q_{2}-p_{2} q_{1}\right) \neq 0$. In order to construct the two cross-stripe solitons, we use $p_{1}=3, p_{2}=-2, q_{1}=3, q_{2}=0$, $\omega_{1}=0, \omega_{2}=0, \alpha=1, \beta=1, \gamma=1$, and $\delta=1$ in Eq. (16). Eq. (16) then becomes

$u=6\left(\ln \left(1+e^{3 x+3 y-\left(\frac{3}{2}+\sqrt{81}\right) t}+e^{-2 x-\sqrt{20} t}+\left(\frac{89+6 \sqrt{5}}{17+6 \sqrt{5}}\right) e^{x+3 y-\left(\frac{3}{2}+\sqrt{20}+\sqrt{81}\right) t}\right)\right)_{x x}$.

The class of solutions given by Eq. (18) corresponds to the two cross-stripe solitons, which are displayed in Figs. 2(a)-(c). The illustrations are produced in the $(x, y)$-plane for $t=-5, t=$ $0, t=5$, respectively. On the other hand, Figs. 2(d)-(f) illustrate the density views of Figs. 2(a)-(c), respectively. It can be perceived from Figs. 2(a)-(c) and Figs. 2(d)-(f) that two solitons intersect with each other in the $(x, y)$-plane. It is also observed from Figs. 2(d)-(f) that one soliton is perpendicular to the $x$-axis and propagates along the negative direction of the axis of 
$x$, and another soliton crosses diagonally in the $(x, y)$-plane. However, they propagate

229

230 independently keeping their original shapes and amplitudes unaltered. On the other hand, for parallel-stripe solitons, the values of $p_{1}, p_{2}, q_{1}$, and $q_{2}$ satisfy $\left(p_{1}-p_{2}\right)\left(q_{1}-q_{2}\right) \neq 0$, but $p_{1} q_{2}-p_{2} q_{1}=0$. As per the mentioned rules, if we take $p_{1}=5, p_{2}=-3, q_{1}=-5, q_{2}=3$, $\omega_{1}=0, \omega_{2}=0, \alpha=1, \beta=1, \gamma=1$, and $\delta=1$ in Eq. (16), it yields

$u=6\left(\ln \left(1+e^{5 x-5 y+\left(\frac{5}{2}-\sqrt{675}\right) t}+e^{-3 x+3 y-\left(\frac{3}{2}+\sqrt{99}\right) t}+\left(\frac{115+3 \sqrt{33}}{25+3 \sqrt{33}}\right) e^{2 x-2 y+(1-15 \sqrt{3}-3 \sqrt{11}) t}\right)\right)_{x x}$.

The solution indicated by Eq. (19) represents the two parallel-stripe solitons. For $t<0$, two parallel-stripe solitons are separated from each other, one with higher amplitude and another with lower amplitude. At $t=0$, these two stripes overlap with one other. For $t>0$, two parallel-stripe solitons are separated further, but the positions of the solitons are found to be reverse than that of the solitons presented for time $t<0$. Thus, the solitons alter their positions at the mentioned times, but the amplitudes of the solitons remain unchanged during propagation. Due to the shake of brevity, the figures are not included here.

\section{Case II: One x-periodic breather}

To construct one $x$-periodic breather wave solutions of Eq. (3), the constraint parameters $a_{i}, b_{i}$, and $\omega_{i}$ need to satisfy the following conditions for Eq. (16) along with Eq. (15):

$a_{1}=a_{2}^{*}=I m_{1}, b_{1}=p_{1}, b_{2}=p_{1}, \omega_{1}=\omega_{2}=0$, and $m_{1} \neq 0, p_{1} \neq 0$.

Here and henceforth ' $*$ ' refers to the complex conjugate of a complex number, where $I^{2}=-1$. If we substitute the values of the constraint parameters as $m_{1}=1, p_{1}=\frac{1}{2}, \omega_{1}=0, \omega_{2}=0$, $\alpha=1, \beta=1, \gamma=1$, and $\delta=1$ into Eq. (16), it turns into the following form:

$u=6\left(\ln \left(1+2 e^{\frac{1}{2} y-\frac{3}{4} t} \cos \left(x+\frac{1}{2} t\right)+13 e^{y-\frac{3}{2} t}\right)\right)_{x x}$

The solution specified by Eq. (21) presents one $x$-periodic breather wave, which is displayed in Figs. 3(a)-(c) at $t=-5, t=0$, and $t=5$, respectively. On the other hand, Figs. 3(d)-(f) illustrate the density views of Figs. 3(a)-(c) for a clear understanding. It can be realized from 
Fig. 3 that with the evolution of time $(t=-5,0,5)$, the positions of $x$-periodic breather are changed from the negative direction of the $y$-axis to its positive direction. However, the shape and amplitude of the $x$-periodic breather wave remain unchanged.

\section{Case III: One xy-periodic breather}

To find one $x y$-periodic breather wave solutions of Eq. (3), the constraint parameters $a_{i}, b_{i}$, and $\omega_{i}$ are prerequisite to satisfy the following conditions for Eq. (16) together with Eq. (15):

$a_{1}=a_{2}=l_{1}, b_{1}=b_{2}^{*}=p_{1}+I q_{1}, \omega_{1}=\omega_{2}=0$, and $l_{1} \neq 0, p_{1} \neq 0, q_{1} \neq 0$

If we take $l_{1}=\frac{1}{3}, p_{1}=\frac{1}{3}, q_{1}=1, \omega_{1}=0, \omega_{2}=0, \alpha=1, \beta=1, \gamma=1$, and $\delta=1$ in Eq. (16), it reduces to the following form:

$u=6\left(\ln \left(1+e^{\left(\frac{1}{3} x+\left(\frac{1}{3}-i\right) y+\left(-\frac{1}{6}+\frac{1}{2} i+\sqrt{\frac{1}{81}+\frac{1}{3} i}\right) t\right)}+e^{\left(\frac{1}{3} x+\left(\frac{1}{3}+i\right) y+\left(-\frac{1}{6}-\frac{1}{2} i-\sqrt{\frac{1}{81}-\frac{1}{3}} i\right) t\right.}\right)+\right.$

$$
\left.\left.\left(\frac{\sqrt{730}-1}{\sqrt{730}-7}\right) e^{\left(\frac{2}{3} x+\frac{2}{3} y+\left(-\frac{1}{3}+\sqrt{\frac{1}{81}+\frac{1}{3} i}-\sqrt{\frac{1}{81}-\frac{1}{3} i}\right) t\right)}\right)\right)_{x x}
$$

The solution specified by Eq. (23) characterizes one $x y$-periodic breather and is displayed in Figs. 4(a) and 4(c). It is interesting to mention here that one $x y$-periodic breather can be changed into one $y$-periodic breather when we consider $p_{1}=0$ and substitute it to the solution $u$ given by Eq. (16). To ensure such behavior, one $y$-periodic breather is illustrated in Figs. 4(b) and 4(d).

\section{Case IV: Lump soliton and rogue wave solutions}

In 1979, Ablowitz and Satsuma proposed a method called "long wave limit method" [58]. With the assist of this method, the lump solitons can be obtained from $N$-soliton solutions after imposing some restrictions on parameters. In order to compute one lump solution of Eq. (3), setting constraint parameters as $b_{1}=m_{1} a_{1}, b_{2}=m_{2} a_{2}, a_{1}=l_{1} \varepsilon, a_{2}=l_{2} \varepsilon$, and $\omega_{1}=\omega_{2}^{*}=$ $\pi i$ to Eq. (15), the function $f_{2}$ can be rearranged as

$f_{2}=\left(\eta_{1} \eta_{2}+A_{12}\right) l_{1} l_{2} \varepsilon^{2}+O\left(\varepsilon^{3}\right)$. 
275 Taking the long-wave limit as $\varepsilon \rightarrow 0$ in Eq. (24) along with Eq. (16), the lump soliton of Eq.

276 (3) can be expressed as follows:

$277 \quad u=\frac{6 \gamma}{\beta}\left(\frac{2}{\eta_{1} \eta_{2}+A_{12}}-\frac{\left(\eta_{1}+\eta_{2}\right)^{2}}{\left(\eta_{1} \eta_{2}+A_{12}\right)^{2}}\right)$,

278 where

$279 \quad A_{12}=\delta\left(m_{1}+m_{2}\right)+2\left(\sqrt{1-m_{1} \delta} \sqrt{1-m_{2} \delta}\right)-2$,

280 and

$281 \quad \eta_{i}=x+m_{i} y-\left(\frac{1}{2} m_{i} \alpha+\sqrt{1+\gamma-m_{i} \delta}\right) t, i=1,2$

282 Now, setting the constraint parameters as $m_{1}=m_{2}^{*}=p_{1}+I q_{1}\left(q_{1} \neq 0\right)$ to Eqs. (25)-(27), we 283 find that the two-solitons will degenerate into one lump soliton. Taking $p_{1}=1, q_{1}=1, \alpha=$ 1, $\beta=1, \gamma=1$, and $\delta=1$ in Eqs. (25)-(27), one can explore one lump-soliton solution. The time evolution 3D plots of lump soliton are exhibited in Figs. 5(a)-(c) at $t=-5, t=0$, and $t=5$, respectively, whereas, Figs. 5(d)-(f) demonstrate the density views of Figs. 5(a)-(c), respectively. It is clearly seen from the figures that the lump has one peak and two troughs. It is also seen from the figures that peak and trough amplitudes of the lump soliton remain unchanged when time increases $(t=-5,0,5)$. But the crest and trough positions of the lump soliton are changed during the propagation in the $(x, y)$-plane. It is pinpoint to mention here that the line rogue wave can be derived from Eq. (3) through the class of solutions given by Eq. (25), if we assume $m_{1}$ and $m_{2}$ as real constants. The mentioned line rogue wave is a rational solution with the process of growth and decay [39]. The constraints for localized waves and their interaction solutions between two-stripe solitons are summarized in Table 1. 
Table 1. The nonlinear localized waves from two-soliton solutions

\begin{tabular}{|c|c|c|}
\hline$N$-soliton & $\begin{array}{l}\text { Types of the localized } \\
\text { waves }\end{array}$ & Constraints \\
\hline \multirow{5}{*}{$N=2$} & Two stripe solitons & $\begin{array}{l}a_{1}=l_{1}, a_{2}=l_{2}, b_{1}=p_{1}, b_{2}=p_{2}, \omega_{1}=\omega_{2}=0 \\
\text { Cross intersection: }\left(l_{1} p_{2}-l_{2} p_{1}\right) \neq 0 \\
\text { Parallel intersection: }\left(l_{1}-l_{2}\right)\left(p_{1}-p_{2}\right) \neq 0 \text {; but } l_{1} p_{2}-l_{2} p_{1}=0\end{array}$ \\
\hline & One $x$-periodic breather & $a_{1}=a_{2}^{*}=I m_{1}, b_{1}=p_{1}, b_{2}=p_{1}, \omega_{1}=\omega_{2}=0$, and $m_{1} \neq 0, p_{1} \neq 0$ \\
\hline & One $y$-periodic breather & $a_{1}=a_{2}=l_{1}, b_{1}=b_{2}^{*}=I q_{1}, \omega_{1}=\omega_{2}=0$, and $l_{1} \neq 0, q_{1} \neq 0$. \\
\hline & One $(x, y)$-periodic breather & $\begin{array}{l}a_{1}=a_{2}=l_{1}, b_{1}=b_{2}^{*}=p_{1}+I q_{1}, \omega_{1}=\omega_{2}=0, \text { and } l_{1} \neq \\
0, p_{1} \neq 0, q_{1} \neq 0 .\end{array}$ \\
\hline & One lump soliton & $\begin{aligned} b_{r} & =m_{r} a_{r}, a_{r}=l_{r} \varepsilon(r=1,2), m_{1}=m_{2}^{*}=p_{1}+I q_{1}, \omega_{1}= \\
\omega_{2}^{*} & =I \pi, \text { and } \varepsilon \rightarrow 0 .\end{aligned}$ \\
\hline
\end{tabular}

297

\subsection{Interaction solutions among three-stripe solitons}

The following subsections discuss the interaction solutions among three-stripe solitons to the $\mathrm{BqE}$ by their graphical illustrations. Interaction solutions among three-stripe solitons, viz. three cross-stripe solitons, two parallel-stripe solitons with other one stripe soliton, three parallel-stripe solitons, one stripe soliton and one periodic breather, and one stripe soliton and one lump soliton can be obtained from three soliton solutions. In order to get such interaction solutions among three-stripe solitons, setting $N=3$ to Eq. (11), one can obtain a function for three-soliton solutions given by

$f_{3}=1+e^{\theta_{1}}+e^{\theta_{2}}+e^{\theta_{3}}+a_{12} e^{\theta_{1}+\theta_{2}}+a_{13} e^{\theta_{1}+\theta_{3}}+a_{23} e^{\theta_{2}+\theta_{3}}+a_{123} e^{\theta_{1}+\theta_{2}+\theta_{3}}$,

where $\theta_{i}$ and $a_{i j}$ are specified by Eqs. (12) and (13), respectively, and the phase shift satisfies the equation $a_{123}=a_{12} a_{23} a_{13}$. Now, plugging Eq. (28) into Eq. (14), the class of three-soliton solutions of Eq. (3) is found in the following form:

311 On restricting certain conditions on the specified parameters and a long-wave limit approach, one can construct several types of localized interaction solutions. Exploration of these solutions is described in detail as follows. 
Here, we would like to interact the two parallel stripe solitons with other one stripe soliton among three-stripe solitons of Eq. (3), the involved constraint parameters $a_{i}, b_{i}$, and $\omega_{i}$ require to maintain the following conditions for Eq. (29):

$a_{i}=p_{i}, b_{i}=q_{i},(i=1,2,3)$, and $\omega_{1}=\omega_{2}=\omega_{3}=0$,

where $p_{i}, q_{i}$ are real constants, $\prod_{i \neq j}\left(p_{i} q_{j}-p_{j} q_{i}\right)=0$, and $\sum_{i \neq j}\left(p_{i} q_{j}-p_{j} q_{i}\right)^{2} \neq 0$.

Taking $p_{1}=3, p_{2}=-2, p_{3}=1, q_{1}=3, q_{2}=2, q_{3}=1, \omega_{1}=0, \omega_{2}=0, \omega_{3}=0, \alpha=1$, $\beta=1, \gamma=1$, and $\delta=1$, one can obtain the interaction solution among two parallel stripe solitons and other one stripe soliton. The time evolution 3D plots of a solution from the class of solutions given by Eq. (29) for $t=-5, t=2$, and $t=5$ is displayed in Figs. 6(a)-(c), whereas Figs. 6(d)-(f) display the respective density views of Figs. 6(a)-(c) for a better perspective. It is seen from Fig. 6 that the two parallel solitons merge at $t=0$. But, two parallel solitons separate and intersect with the remaining one soliton normally at $t=-5$ as well as at $t=5$. If the constraint parameters satisfy the condition $\prod_{i \neq j}\left(p_{i} q_{j}-p_{j} q_{i}\right) \neq 0$ for Eq. (28), the solution obtained from Eq. (29) represents three cross-stripe solitons. Alternatively, if the constraint parameters satisfy the condition $\sum_{i \neq j}\left(p_{i} q_{j}-p_{j} q_{i}\right)^{2}=0$ for Eq. (28), the class of solutions given by Eq. (29) represents three parallel-stripe solitons. Due to the sake of conciseness, three cross-stripe and three parallel-stripe solitons are not displayed here. For three cross-stripe solitons, the selected parameters are $p_{1}=3, p_{2}=2, p_{3}=1, q_{1}=3, q_{2}=$ $-2, q_{3}=-0.5, \omega_{1}=0, \omega_{2}=0, \omega_{3}=0$, and $p_{1}=3, p_{2}=2, p_{3}=1, q_{1}=3, q_{2}=2, q_{3}=$ 1, $\omega_{1}=0, \omega_{2}=0, \omega_{3}=0$ are selected for three parallel-stripe solitons. For both cases, $\alpha=$ $1, \beta=1, \gamma=1$, and $\delta=1$ are used. 


\section{Case II: One stripe soliton and one periodic breather}

340

341

342

To consider the interaction solutions between one stripe soliton and one $(x, y)$-periodic breather wave for Eq. (3), the constraint parameters $a_{i}, b_{i}$, and $\omega_{i}$ allow to maintain the following conditions for Eq. (29):

$a_{1}=l_{1}+I m_{1}, a_{2}=l_{1}-I m_{1}, b_{1}=p_{1}+I q_{1}, b_{2}=p_{1}-I q_{1}, a_{3}=l_{2}, b_{3}=p_{2}$, and $\omega_{1}=$

$\omega_{2}=\omega_{3}=0$

where $l_{1} \neq 0, l_{2} \neq 0, m_{1} \neq 0, p_{1} \neq 0, p_{2} \neq 0$, and $q_{1} \neq 0$.

It is mentionable at this juncture that for the parallel one stripe soliton and one $(x, y)$-periodic breather wave, the constraint parameters must follow the condition $l_{1} p_{2}-l_{2} p_{1}=0$, and for the cross one stripe soliton and one $(x, y)$-periodic breather wave, the constraint parameters must satisfy the condition $l_{1} p_{2}-l_{2} p_{1} \neq 0$. Now, if we set the constraint parameters as $a_{1}=$ $a_{2}=0.25, a_{3}=-0.75, b_{1}=b_{2}^{*}=-0.25+0.75 I, b_{3}=0.75, \omega_{1}=0, \omega_{2}=0, \omega_{3}=0$, $\alpha=1, \beta=1, \gamma=1$, and $\delta=1$ to Eq. (29), it corresponds to the interaction solution between the parallel one stripe soliton and one $(x, y)$-periodic breather wave. The time evolution density graphs of the respective solution at time $t=-5, t=0$, and $t=5$ are demonstrated through Figs. 7(a1)-(a3). At time $t=-5$, one stripe soliton and one $(x, y)$-periodic breather waves are separated (see Fig. 7(a1)). Then, at time $t=0$, they are merged together (see Fig. 7(a2)). Finally, at time $t=5$, they are separated again. During the propagation, the amplitudes of the soliton and breather remain unchanged. The interaction behaviors of stripe and breather waves are elastic. It is worthy of note here that if we set $l_{1}=0, q_{1}=0$ to the condition given by Eq. (31), the interaction between one stripe soliton and one $(x, y)$-periodic breather reforms to the interaction between one stripe soliton and one $x$-periodic breather. By selecting the constraint parameters as $a_{1}=a_{2}^{*}=0.25 I, a_{3}=-0.75, b_{1}=b_{2}=-0.25, b_{3}=0.75, \omega_{1}=0, \omega_{2}=0$, $\omega_{3}=0, \alpha=1, \beta=1, \gamma=1$, and $\delta=1$ to the condition specified by Eq. (31), the time evolution density graphs of one stripe soliton and one $x$-periodic breather are presented in Figs. 


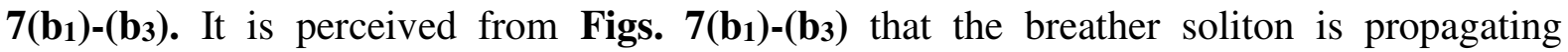
parallelly along the $x$-axis. Furthermore, if we set $m_{1}=0, p_{1}=0$ to the condition given by Eq. (31), the collision between one stripe soliton and one $(x, y)$-periodic breather turns into the collision between one stripe soliton and one $y$-periodic breather. Setting the constraint parameters $a_{1}=a_{2}=0.25, a_{3}=-0.75, b_{1}=b_{2}^{*}=0.75 \mathrm{I}, b_{3}=0.75, \omega_{1}=0, \omega_{2}=0$, $\omega_{3}=0, \alpha=1, \beta=1, \gamma=1$, and $\delta=1$ to Eq. (29), one can produce the interaction solution between one stripe soliton and one $y$-periodic breather. The time evolution density graphs of

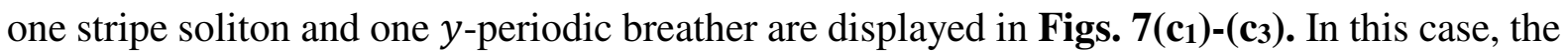
corresponding figures show that the breather wave propagates parallelly along the $y$-axis. It can be observed from each panel of density graphs that with the evolution of time, the stripe and all periodic breathers change their positions retaining their amplitudes unchanged.

\section{Case III: One lump soliton and one stripe soliton}

In order to detect the interaction solutions between one lump soliton and one stripe soliton for Eq. (3), the constraint parameters $a_{i}, b_{i}$, and $\omega_{i}$ require to maintain the following conditions for Eq. (29) in consort with Eq. (28):

$$
b_{1}=m_{1} a_{1}, b_{2}=m_{2} a_{2}, b_{3}=m_{3} a_{3}, a_{1}=l_{1} \varepsilon, a_{2}=l_{2} \varepsilon, \omega_{1}=\omega_{2}^{*}=I \pi, \text { and } \omega_{3}=0 .
$$

Inserting the values presented in Eq. (32) into Eq. (28), it is possible to present the function $f_{3}$ as

$f_{3}=\left(A_{12}+\eta_{1} \eta_{2}\right) l_{1} l_{2} \varepsilon^{2}+\left(\eta_{1} \eta_{2}+A_{23} \eta_{1}+A_{13} \eta_{2}+A_{12}+A_{12} A_{23}\right) e^{\theta_{3}} l_{1} l_{2} \varepsilon^{2}+O\left(\varepsilon^{3}\right)$.

Taking the long-wave limit as $\varepsilon \rightarrow 0$ in Eq. (33), the class of interaction solutions between one lump and one stripe soliton of Eq. (3) can be obtained from Eq. (29). This leads to

$$
u=\frac{6 \gamma}{\beta}\left(\ln \left(\left(A_{12}+\eta_{1} \eta_{2}\right)+\left(\eta_{1} \eta_{2}+A_{23} \eta_{1}+A_{13} \eta_{2}+A_{12}+A_{12} A_{23}\right) e^{\theta_{3}}\right)\right)_{x x},
$$

where

$$
A_{12}=\delta\left(m_{1}+m_{2}\right)+2\left(\sqrt{1-m_{1} \delta} \sqrt{1-m_{2} \delta}\right)-2,
$$


$\eta_{i}=x+m_{i} y-\left(\frac{1}{2} m_{i} \alpha+\sqrt{1+\gamma-m_{i} \delta}\right) t, i=1,2$

and

$\theta_{3}=a_{3} x+m_{3} a_{3} y-\left(\frac{1}{2} \alpha m_{3} a_{3}+\sqrt{\gamma a_{3}^{4}-\delta m_{3} a_{3}^{2}+a_{3}^{2}}\right) t$

Setting the constraint parameters $m_{1}=m_{2}^{*}=1+I, m_{3}=-0.5$, and $a_{3}=0.5$ to Eqs. (34)(38), one can see the elastic interaction between one lump soliton and one stripe soliton in Fig.

8. Figs. 8(a1)-8(as) illustrate the $3 \mathrm{D}$ plots in the $(x, y)$-plane at $t=-20, t=-10, t=0, t=$ 10, $t=20$, respectively, whereas Figs. 8( $\left(\mathbf{b}_{1}\right)-8\left(\mathbf{b}_{5}\right)$ demonstrate the density views of Figs. 8(a1)-8(a5), respectively. At $t=-20$, lump soliton appears in the negative side of the $x$-axis and is separated from the line soliton (see Fig. 8(a1)). It can be also seen from Figs. 8(a2)-(a3) that the lump soliton is gradually integrated with the soliton as time goes on $(t=-10,0)$. Furthermore, as the time increases $(t=10,20)$, the lump soliton gradually appears on the positive side of the $x$-axis and is separated from the line soliton, which can clearly be observed from Figs. 8(a4)-(a5). During the propagation, the amplitude of the lump soliton is found to be unchanged. The mentioned behaviors can be clarified from the corresponding density views displayed in Figs. 8(b1)-8(b5).

The overview of restrictions of parameters for localized waves and their interaction solutions among three solitons of Eq. (3) are presented in Table 2. The first column in Table 2 shows the order of $N$-soliton, while the second column shows the types of localized wave solutions. localized wave solutions. 
Table 2. The nonlinear localized wave interaction structures of $N$-soliton solutions

\begin{tabular}{|c|c|c|}
\hline$N$-soliton & $\begin{array}{l}\text { Localized waves and } \\
\text { interaction structures }\end{array}$ & Constraints \\
\hline \multirow{9}{*}{$N=3$} & \multirow{5}{*}{ Three stripe solitons } & $a_{i}=p_{i}, b_{i}=q_{i},(i=1,2,3)$, and $\omega_{1}=\omega_{2}=\omega_{3}=0$ \\
\hline & & For cross intersection: $\prod_{i \neq j}\left(p_{i} q_{j}-p_{j} q_{i}\right) \neq 0$ \\
\hline & & For parallel intersection: $\sum_{i \neq j}\left(p_{i} q_{j}-p_{j} q_{i}\right)^{2}=0$ \\
\hline & & For cross and parallel intersection: \\
\hline & & $\prod_{i \neq j}\left(p_{i} q_{j}-p_{j} q_{i}\right)=0$, and $\sum_{i \neq j}\left(p_{i} q_{j}-p_{j} q_{i}\right)^{2} \neq 0$ \\
\hline & $\begin{array}{l}\text { One stripe soliton + one } x \text { - } \\
\text { periodic breather }\end{array}$ & $\begin{array}{l}a_{1}=a_{2}^{*}=I m_{1}, b_{1}=b_{2}=p_{1}, a_{3}=m_{2}, b_{3}=p_{2}, \text { and } \omega_{1}=\omega_{2}= \\
\omega_{3}=0\end{array}$ \\
\hline & $\begin{array}{l}\text { One stripe soliton }+ \text { one } y \text { - } \\
\text { periodic breather }\end{array}$ & $\begin{array}{l}a_{1}=a_{2}=l_{1}, b_{1}=b_{2}^{*}=I q_{1}, a_{3}=l_{2}, b_{3}=q_{2}, \text { and } \omega_{1}=\omega_{2}=\omega_{3}= \\
0 .\end{array}$ \\
\hline & $\begin{array}{l}\text { One stripe soliton + one }(x, y) \text { - } \\
\text { periodic breather }\end{array}$ & $\begin{array}{l}a_{1}=a_{2}=l_{1}, b_{1}=b_{2}^{*}=p_{1}+I q_{1}, a_{3}=l_{2}, b_{3}=p_{2}, \text { and } \omega_{1}=\omega_{2}= \\
\omega_{3}=0 . \\
\text { For cross intersection: } l_{1} p_{2}-l_{2} p_{1} \neq 0, \text { and } \\
\text { For parallel intersection: } l_{1} p_{2}-l_{2} p_{1}=0 .\end{array}$ \\
\hline & $\begin{array}{l}\text { One stripe soliton + one lump } \\
\text { soliton }\end{array}$ & $\begin{array}{l}b_{1}=m_{1} a_{1}, b_{2}=m_{2} a_{2}, b_{3}=m_{3} a_{3}, a_{1}=l_{1} \varepsilon, a_{2}=l_{2} \varepsilon, m_{1}=m_{2}^{*}= \\
p_{1}+I q_{1}, \omega_{1}=\omega_{2}^{*}=I \pi, \omega_{3}=0, \text { and } \varepsilon \rightarrow 0 .\end{array}$ \\
\hline
\end{tabular}

412

\subsection{Interaction solutions among four-stripe solitons}

The following subsections discuss the interaction solutions among four-stripe solitons to the $\mathrm{BqE}$ by their graphical illustrations. To reach such aims, setting $N=4$ to Eq. (11), one can attain a function for four-soliton solutions given by

$f_{4}=1+e^{\theta_{1}}+e^{\theta_{2}}+e^{\theta_{3}}+e^{\theta_{4}}+a_{12} e^{\theta_{1}+\theta_{2}}+a_{13} e^{\theta_{1}+\theta_{3}}+a_{14} e^{\theta_{1}+\theta_{4}}+a_{23} e^{\theta_{2}+\theta_{3}}+$

$+a_{24} e^{\theta_{2}+\theta_{4}}+a_{34} e^{\theta_{3}+\theta_{4}}+a_{123} e^{\theta_{1}+\theta_{2}+\theta_{3}}+a_{124} e^{\theta_{1}+\theta_{2}+\theta_{4}}+a_{134} e^{\theta_{1}+\theta_{3}+\theta_{4}}+$

$a_{234} e^{\theta_{2}+\theta_{3}+\theta_{4}}+a_{1234} e^{\theta_{1}+\theta_{2}+\theta_{3}+\theta_{4}}$,

where $\theta_{i}$ and $a_{i j}$ are specified by Eqs. (12) and (13), respectively, and the phase shifts follow

$a_{123}=a_{12} a_{23} a_{13}$ and $a_{1234}=a_{12} a_{13} a_{14} a_{23} a_{24} a_{34}$. form: 
On restricting certain conditions on the given parameters and a long-wave limit approach, one can achieve several types of localized interaction solutions. The construction procedure of the localized interaction solutions is described as follows:

\section{Case I: Interaction among two-stripe solitons and one periodic breather}

For Eq. (40), if we take $a_{1}=a_{2}^{*}=l_{1}+I m_{1}, b_{1}=b_{2}^{*}=p_{1}+I q_{1}, a_{3}=l_{2}, a_{4}=l_{3}, b_{3}=p_{2}$, $b_{4}=p_{3}$, and $\omega_{1}=\omega_{2}=\omega_{3}=\omega_{4}=0$, the four-stripe soliton solutions will degenerate into the interaction among two-stripe solitons and one periodic breather wave solutions. For different values of $l_{1}, m_{1}, p_{1}, q_{1}, l_{2}, l_{3}, p_{2}$, and $p_{3}$, the periodic breather can move along a line parallel to the $x$ or $y$ axes or its cross-sectional direction. More specifically, three assumptions can be made, such as the interaction among (i) two-stripe solitons and one $x$-periodic breather,

(ii) two-stripe solitons and one $y$-periodic breather, and (iii) two-stripe solitons and one $x y$ periodic breather. Figs. 9(a)-(f) present such types of interaction solutions in the $(x, y)$-plane.

(i) Setting $a_{1}=a_{2}^{*}=0.50 I, a_{3}=0.50, a_{4}=0.75, b_{1}=b_{2}=0.25, b_{3}=0.25, b_{4}=0.75$, $\omega_{1}=0, \omega_{2}=0, \omega_{3}=0, \omega_{4}=0, \alpha=1, \beta=1, \gamma=1$, and $\delta=1$ in the class of solutions given by Eq. (40), one can characterize the interaction solution among two-stripe solitons and one $x$-periodic breather. Such characteristics are illustrated by their 3D and respective density views displayed in Figs. 9(a) and 9(d), respectively. It is seen from the imposing constraint values that the breather wave is parallel to the $x$-axis when $a_{1}$ and $a_{2}$ are purely imaginary numbers and the others are real numbers.

(ii) Substituting $a_{1}=a_{2}=0.25, a_{3}=0.25, a_{4}=0.50, b_{1}=b_{2}^{*}=0.45 I, b_{3}=0.50, b_{4}=$ $0.50, \omega_{1}=0, \omega_{2}=0, \omega_{3}=0, \omega_{4}=0, \alpha=1, \beta=1, \gamma=1$, and $\delta=1$ in the class of solutions specified by Eq. (40), one can represent the interaction solutions among two-stripe solitons and one $y$-periodic breather. These behaviors are exhibited by their 3D and density plots through Fig. 9(b) and Fig. 9(c), respectively. When $b_{1}$ and $b_{2}$ are purely imaginary numbers, and the others are real numbers, the breather wave is periodic only along the $y$-axis. 
(iii) Putting $a_{1}=a_{2}=\frac{1}{3}, a_{3}=\frac{1}{3}, a_{4}=\frac{1}{3}, b_{1}=b_{2}^{*}=-0.3+I, b_{3}=0.8, b_{4}=0.25, \omega_{1}=0$, $\omega_{2}=0, \omega_{3}=0, \omega_{4}=0, \alpha=1, \beta=1, \gamma=1$, and $\delta=1$ in the class of solutions given by Eq. (40), one can expose the interaction solution among two-stripe solitons and one $y$-periodic breather. These behaviors are exposed by their 3D and density plots through Figs. 9(c) and 9(e), respectively. In such cases, $b_{1}, b_{2}$ are imaginary numbers and the remaining are real numbers.

\section{Case II: Interaction between two periodic breathers}

457

The class of solutions presented by Eq. (40) expresses the interaction between two parallel $x$ periodic breathers, when we set the constraint parameters as $a_{1}=a_{2}^{*}=0.50 I, a_{3}=a_{4}^{*}=$ $0.50 I, b_{1}=b_{2}=0.3, b_{3}=b_{4}=0.50, \omega_{1}=0, \omega_{2}=0, \omega_{3}=0, \omega_{4}=0, \alpha=1, \beta=1, \gamma=$ 1, and $\delta=1$. Two parallel $x$-periodic breathers are displayed in Figs. 10(a) and 10(d) through the 3D and its density graphs. The interaction between two parallel $x$-periodic breathers turns into two parallel $y$-periodic breathers when we set $a_{1}=a_{2}=0.25, a_{3}=a_{4}=0.50, b_{1}=$ $b_{2}^{*}=-0.50 I, b_{3}=b_{4}^{*}=0.50 I, \omega_{1}=0, \omega_{2}=0, \omega_{3}=0, \omega_{4}=0, \alpha=1, \beta=1, \gamma=1$, $\delta=1$ in Eq. (40). These behaviors are illustrated in Figs. 10(b) and 10(e). Now, considering $a_{1}=a_{2}=0.35, a_{3}=a_{4}=0.2, b_{1}=b_{2}^{*}=0.35-0.50 I, b_{3}=b_{4}^{*}=-0.25+0.75 I, \omega_{1}=$ $0, \omega_{2}=0, \omega_{3}=0, \omega_{4}=0, \alpha=1, \beta=1, \gamma=1, \delta=1$ in Eq. (40), one can attain the interaction between two cross $x y$-periodic breathers as shown in Figs. 10(c) and 10(f). It can be observed from the above figures that two breathers retain their velocities and shapes unchanged during propagation. The propagation behavior is not displayed due to the sake of conciseness. However, it is not so difficult to perceive that their interaction is elastic. That means, two periodic breathers only exchange their positions after this elastic collision procedure. 
476

477

478

479

480

481

482

483

484

485

486

487

488

489

490

491

492

493

494

495

496

In order to explore the interaction solutions among one lump soliton and one periodic breather, and one lump soliton and two solitons for Eq. (3), the constraint parameters $a_{i}, b_{i}$, and $\omega_{i}$ guarantee to satisfy the following conditions for Eq. (40) along with Eq. (39):

$$
b_{i}=m_{i} a_{i}(i=1,2,3,4), a_{1}=l_{1} \varepsilon, a_{2}=l_{2} \varepsilon, \omega_{1}=\omega_{2}^{*}=I \pi, \text { and } \omega_{3}=\omega_{4}=0 .
$$

Inserting the values provided by Eq. (41) into Eq. (39), one can rewrite the function $f_{4}$ as follows:

$f_{4}=\left(A_{12}+\eta_{1} \eta_{2}\right) l_{1} l_{2} \varepsilon^{2}+\left(\eta_{1} \eta_{2}+A_{23} \eta_{1}+A_{13} \eta_{2}+A_{12}+A_{13} A_{23}\right) e^{\theta_{3}} l_{1} l_{2} \varepsilon^{2}+\left(\eta_{1} \eta_{2}+\right.$ $\left.A_{24} \eta_{1}+A_{14} \eta_{2}+A_{12}+A_{14} A_{24}\right) e^{\theta_{4}} l_{1} l_{2} \varepsilon^{2}+A_{34}\left[\eta_{1} \eta_{2}+\left(A_{23}+A_{24}\right) \eta_{1}+\left(A_{13}+A_{14}\right) \eta_{2}+\right.$

$\left.A_{12}+\left(A_{13}+A_{14}\right)\left(A_{23}+A_{24}\right)\right] \times e^{\left(\theta_{3}+\theta_{4}\right)} l_{1} l_{2} \varepsilon^{2}+O\left(\varepsilon^{3}\right)$.

Taking the long-wave limit as $\varepsilon \rightarrow 0$ in Eq. (42), the class of interaction solutions between one lump and one stripe soliton of Eq. (3) can be obtained from Eq. (40), which is given by

$u=\frac{6 \gamma}{\beta}\left(\ln \left(\left(A_{12}+\eta_{1} \eta_{2}\right)+\left(\eta_{1} \eta_{2}+A_{23} \eta_{1}+A_{13} \eta_{2}+A_{12}+A_{13} A_{23}\right) e^{\theta_{3}}+\left(\eta_{1} \eta_{2}+\right.\right.\right.$

$\left.A_{24} \eta_{1}+A_{14} \eta_{2}+A_{12}+A_{14} A_{24}\right) e^{\theta_{4}}+A_{34}\left[\eta_{1} \eta_{2}+\left(A_{23}+A_{24}\right) \eta_{1}+\left(A_{13}+A_{14}\right) \eta_{2}+A_{12}+\right.$

$\left.\left.\left.\left(A_{13}+A_{14}\right)\left(A_{23}+A_{24}\right)\right] \times e^{\left(\theta_{3}+\theta_{4}\right)}\right)\right)_{x x}$,

where

$\eta_{i}=x+m_{i} y-\left(\frac{1}{2} m_{i} \alpha+\sqrt{1+\gamma-m_{i} \delta}\right) t, i=1,2$

$A_{12}=\delta\left(m_{1}+m_{2}\right)+2\left(\sqrt{1-m_{1} \delta} \sqrt{1-m_{2} \delta}\right)-2$,

$A_{k r}=-2 a_{r}-4 \gamma a_{r}^{3}+\delta a_{r} m_{k}+\delta m_{r} a_{r}+2\left(\sqrt{1-m_{k} \delta} \sqrt{a_{r}^{2}\left(\gamma a_{r}^{2}-\delta m_{r}+1\right)}\right),(k=1,2 ; r=3,4)$,

$A_{34}=\frac{2+4 \gamma a_{3}^{2}-6 \gamma a_{3}^{2} a_{4}+4 \gamma a_{4}^{2}+\delta m_{4}+\delta m_{4}-2\left(\sqrt{\left(\gamma a_{3}^{2}-\delta m_{3}+1\right)} \sqrt{\left(\gamma a_{4}^{2}-\delta m_{4}+1\right)}\right)}{2+4 \gamma a_{3}^{2}+6 \gamma a_{3}^{2} a_{4}+4 \gamma a_{4}^{2}+\delta m_{4}+\delta m_{4}-2\left(\sqrt{\left(\gamma a_{3}^{2}-\delta m_{3}+1\right)} \sqrt{\left(\gamma a_{4}^{2}-\delta m_{4}+1\right)}\right)}$,

and

$\theta_{i}=a_{i} x+m_{i} a_{i} y-\left(\frac{1}{2} \alpha m_{i} a_{i}+\sqrt{\gamma a_{i}^{4}-\delta m_{i} a_{i}^{2}+a_{i}^{2}}\right) t, i=3,4$ 
(i) Considering $m_{1}=m_{2}^{*}=p_{1}+I q_{1}, m_{3}=m_{4}^{*}=p_{2}+I q_{2}$, and $a_{3}=a_{4}=\vartheta_{1}$ in Eqs. (43)-

498

499

500

501

502

503

504

505

506

507

508

509

510

511

512

513

514

515

516

517

518

519

520

521

(48), the class of solutions specified by Eq. (43) expresses the interaction solutions between one lump and one breather wave. In particular, taking $p_{1}=0.5, q_{1}=1.5, p_{2}=0, q_{2}=0.75$, $\vartheta_{1}=-0.25, \alpha=1, \beta=1, \gamma=1, \delta=1$, the specific structures of the interaction between one lump and one breather are displayed in Fig. 11. Figs. 11(a1)-(as) illustrate the 3D plots of the specific solution obtained from Eq. (43) at $t=-40, t=-20, t=0, t=20, t=40$, respectively, whereas Figs. 11(b1)-(b5) display the corresponding density views of Figs. 11(a1)-(a5). At time $t=-40$, the lump soliton is found on the negative side of the $x$-axis, while the breather is found on the positive side of the $x$-axis. With the evolution of time ( $t=$ $-20,0,20,40)$, both breather and lump soliton propagate and change their positions. Thus, it is clear from the wave propagation behaviors (Figs. 11(a1)-(a5) or Figs. 11(b1)-(b5)) that the interaction structures among the solitons mentioned above is completely elastic.

(ii) When selecting $m_{1}=m_{2}^{*}=p_{1}+I q_{1}, m_{3}=p_{2}, m_{4}=p_{3}$, and $a_{3}=a_{4}=\vartheta_{1}$ to Eqs. (43)-(48), the solution given by Eq. (43) is reduced to the interaction among one lump and twostripe solitons. Taking the particular values of the constraint parameters as $p_{1}=0.75, q_{1}=$ $0.75, p_{2}=-2, p_{3}=-1, \vartheta_{1}=-0.6, \alpha=1, \beta=1, \gamma=1, \delta=1$, the aforesaid interaction soliton solution is displayed in Fig. 12. The interaction among the solitons is also elastic. It is remarkable to mention here that if we set $p_{1}=0.75, q_{1}=0.75, p_{2}=-1, p_{3}=-1, \vartheta_{1}=$ $-0.6, \alpha=1, \beta=1, \gamma=1, \delta=1$ in the solution prescribed by Eq. (43), it turns into the interaction between one lump and one-stripe solitons.

\section{Case IV: Interaction between two lump solitons}

In order to search the interaction solutions between two lump solitons for Eq. (3), the constraint parameters $a_{i}, b_{i}$, and $\omega_{i}$ allow to satisfy the following conditions for Eq. (40) along with Eq. (39):

$$
b_{i}=m_{i} a_{i}(i=1,2,3,4), a_{i}=l_{i} \varepsilon(i=1,2,3,4), \omega_{1}=\omega_{2}^{*}=I \pi \text {, and } \omega_{3}=\omega_{4}^{*}=I \pi \text {. }
$$


Substituting the values provided by Eq. (49) into Eq. (39), the function $f_{4}$ can be brought as follows:

$f_{4}=\left(\eta_{1} \eta_{2} \eta_{3} \eta_{4}+A_{34} \eta_{1} \eta_{2}+A_{24} \eta_{1} \eta_{3}+A_{23} \eta_{1} \eta_{4}+A_{14} \eta_{2} \eta_{3}+A_{13} \eta_{2} \eta_{4}+A_{12} \eta_{3} \eta_{4}+\right.$ $\left.A_{12} A_{34}+A_{13} A_{24}+A_{14} A_{23}\right) l_{1} l_{2} l_{3} l_{4} \varepsilon^{4}+O\left(\varepsilon^{5}\right)$.

Taking the long-wave limit as $\varepsilon \rightarrow 0$ in Eq. (50), the interaction between one lump and one stripe soliton solutions of Eq. (3) can be obtained from Eq. (40), which leads to $u=\frac{6 \gamma}{\beta}\left(\ln \left(\eta_{1} \eta_{2} \eta_{3} \eta_{4}+A_{34} \eta_{1} \eta_{2}+A_{24} \eta_{1} \eta_{3}+A_{23} \eta_{1} \eta_{4}+A_{14} \eta_{2} \eta_{3}+A_{13} \eta_{2} \eta_{4}+A_{12} \eta_{3} \eta_{4}+\right.\right.$ $\left.\left.A_{12} A_{34}+A_{13} A_{24}+A_{14} A_{23}\right)\right)_{x x}$

where

$\eta_{i}=x+m_{i} y-\left(\frac{1}{2} m_{i} \alpha+\sqrt{1+\gamma-m_{i} \delta}\right) t, i=1,2,3,4$

$A_{i j}=\delta\left(m_{i}+m_{j}\right)+2\left(\sqrt{1-m_{i} \delta} \sqrt{1-m_{j} \delta}\right)-2,(1 \leq i \leq j \leq 4)$.

The class of solutions indicated by Eq. (51) expresses two lump solitons. Considering $m_{1}=$ $m_{2}^{*}=-1+I, m_{3}=m_{4}^{*}=-1+10 I, \alpha=1, \beta=1, \gamma=1, \delta=1$ in Eq. (51), the propagation and time evolution of $3 \mathrm{D}$ plots of the elastic interaction between two lump solitons are shown in Figs. 13(a)-13(f) for time variation $t=-50, t=-25, t=0, t=25, t=50$, respectively. At $t=-50$, two lump solitons appear on the negative side of the $x$-axis with different amplitudes (see Fig. 13(a)). With the evolution of time $(t=-25,0,25,50)$, two lump solitons propagate from the negative side of the $x$-axis to its positive side, which is clearly realized from Figs. 13(b)-(f). It can be visualized from Figs. 13(a)-(f) that the two lumps propagate with the same velocity along the $x$-axis. During the propagation, the two lumps get closer, then collide with each other, and finally move apart. It is also perceived from the figures that during the propagation, two lumps preserve their amplitudes, but they altered their positions. The overview of the constraint parameters for the foursoliton solutions of Eq. (3) is presented in Table 3. The first column in Table 3 shows the order of $\mathrm{N}$ soliton, the second column shows the localized wave structures, and the last column provides the corresponding parameters of the localized wave interaction solutions in the second column. 
Table 3. The nonlinear localized wave interaction structures of $N$-soliton solutions

\begin{tabular}{|c|c|c|}
\hline$N$-soliton & $\begin{array}{l}\text { Localized waves and } \\
\text { interaction structures }\end{array}$ & Constraints \\
\hline \multirow{11}{*}{$N=4$} & $\begin{array}{l}\text { Two stripe solitons }+ \text { one } y \text { - } \\
\text { periodic breather }\end{array}$ & $\begin{array}{l}a_{1}=a_{2}=l_{1}, b_{1}=b_{2}^{*}=I q_{1}, a_{3}=l_{2}, a_{4}=l_{3}, b_{3}=p_{2}, b_{4}=p_{3}, \text { and } \\
\omega_{1}=\omega_{2}=\omega_{3}=\omega_{4}=0 .\end{array}$ \\
\hline & $\begin{array}{l}\text { Two stripe solitons }+ \text { one } x \text { - } \\
\text { periodic breather }\end{array}$ & $\begin{array}{l}a_{1}=a_{2}^{*}=I m_{1}, b_{1}=b_{2}=p_{1}, a_{3}=m_{2}, a_{4}=m_{3}, b_{3}=p_{2}, b_{4}=p_{3}, \\
\text { and } \omega_{1}=\omega_{2}=\omega_{3}=\omega_{4}=0\end{array}$ \\
\hline & $\begin{array}{l}\text { Two stripe solitons }+ \text { one } \\
(x, y) \text {-periodic breather }\end{array}$ & $\begin{array}{l}a_{1}=a_{2}=l_{1}, b_{1}=b_{2}^{*}=p_{1}+I q_{1}, a_{3}=l_{2}, a_{4}=l_{3}, b_{3}=p_{2}, b_{4}=p_{3} \text {, } \\
\text { and } \omega_{1}=\omega_{2}=\omega_{3}=\omega_{4}=0 \text {. }\end{array}$ \\
\hline & $\begin{array}{l}\text { Two parallel } y \text {-periodic } \\
\text { breathers }\end{array}$ & $\begin{array}{l}a_{1}=a_{2}=l_{1}, b_{1}=b_{2}^{*}=I q_{1}, a_{3}=a_{4}=l_{2}, b_{3}=b_{4}^{*}=I q_{2}, \text { and } \omega_{1}= \\
\omega_{2}=\omega_{3}=\omega_{4}=0 .\end{array}$ \\
\hline & $\begin{array}{l}\text { Two parallel } x \text {-periodic } \\
\text { breathers }\end{array}$ & $\begin{array}{l}a_{1}=a_{2}^{*}=I m_{1}, b_{1}=b_{2}=p_{1}, a_{3}=a_{4}^{*}=I m_{2}, b_{3}=b_{4}=p_{2}, \text { and } \omega_{1}= \\
\omega_{2}=\omega_{3}=\omega_{4}=0 .\end{array}$ \\
\hline & $\begin{array}{l}\text { Two cross }(x, y) \text {-periodic } \\
\text { breathers }\end{array}$ & $\begin{array}{l}a_{1}=a_{2}=l_{1}, b_{1}=b_{2}^{*}=p_{1}+I q_{1}, a_{3}=a_{4}=l_{2}, b_{3}=b_{4}^{*}=p_{2}+I q_{2} \\
\text { and } \omega_{1}=\omega_{2}=\omega_{3}=\omega_{4}=0\end{array}$ \\
\hline & $\begin{array}{l}\text { One } y \text {-periodic breather }+ \text { one } \\
(x, y) \text {-periodic breather }\end{array}$ & $\begin{array}{l}a_{1}=a_{2}=l_{1}, b_{1}=b_{2}^{*}=I q_{1}, a_{3}=a_{4}=l_{2}, b_{3}=b_{4}^{*}=p_{2}+I q_{2}, \text { and } \\
\omega_{1}=\omega_{2}=\omega_{3}=\omega_{4}=0 .\end{array}$ \\
\hline & $\begin{array}{l}\text { One } x \text {-periodic breather }+ \text { one } \\
(x, y) \text {-periodic breather }\end{array}$ & $\begin{array}{l}a_{1}=a_{2}^{*}=I m_{1}, b_{1}=b_{2}=p_{1}, a_{3}=a_{4}=m_{2}, b_{3}=b_{4}^{*}=p_{2}+I q_{2}, \text { and } \\
\omega_{1}=\omega_{2}=\omega_{3}=\omega_{4}=0\end{array}$ \\
\hline & $\begin{array}{l}\text { One } x \text {-periodic breather }+ \text { one } \\
y \text {-periodic breather }\end{array}$ & $\begin{array}{l}a_{1}=a_{2}^{*}=I m_{1}, b_{1}=b_{2}=p_{1}, a_{3}=a_{4}=m_{2}, b_{3}=b_{4}^{*}=I q_{2}, \text { and } \omega_{1}= \\
\omega_{2}=\omega_{3}=\omega_{4}=0 .\end{array}$ \\
\hline & Two lump solitons & $\begin{array}{l}b_{1}=m_{1} a_{1}, b_{2}=m_{2} a_{2}, b_{3}=m_{3} a_{3}, b_{4}=m_{4} a_{4}, a_{1}=l_{1} \varepsilon_{1}, a_{2}=l_{2} \varepsilon_{1}, \\
a_{3}=l_{3} \varepsilon_{2}, a_{4}=l_{4} \varepsilon_{2}, \omega_{1}=\omega_{2}^{*}=\omega_{3}=\omega_{4}^{*}=I \pi, m_{1}=m_{2}^{*}=p_{1}+I q_{1}, \\
m_{3}=m_{4}^{*}=p_{2}+I q_{2}, \varepsilon_{1} \rightarrow 0, \text { and } \varepsilon_{2} \rightarrow 0 .\end{array}$ \\
\hline & $\begin{array}{l}\text { One lump + one periodic } \\
\text { breather }\end{array}$ & $\begin{array}{l}b_{1}=m_{1} a_{1}, b_{2}=m_{2} a_{2}, a_{3}=a_{4}=\alpha_{2}, b_{3}=b_{4}^{*}=p_{2}+I q_{2}, a_{1}=l_{1} \varepsilon, \\
a_{2}=l_{2} \varepsilon, \omega_{1}=\omega_{2}^{*}=I \pi, \omega_{3}=\omega_{4}=0, m_{1}=m_{2}^{*}=p_{1}+I q_{1}, \text { and } \varepsilon \rightarrow \\
0 .\end{array}$ \\
\hline
\end{tabular}

\section{Discussion of the results and concluding remarks}

It is mentioned earlier in the introduction section that Wazwaz and Kaur [52] examined a family of BqEs with distinct dimensions first time. They reported some real and complex multiple soliton solutions by virtue of the simplified Hirota's method. In addition, they also reported a diverse range of soliton solutions in terms of hyperbolic, trigonometric, and rational functions via the exponential expansion scheme. However, in this article, we have used a dependent variable transformation to a dimensionally reduced $(2+1)$-dimensional $\mathrm{BqE}$ and thereby constructed $N$-soliton solutions via the HBM. Afterward, via the long-wave limit 
approach in coordination with certain conditions on the parameters associated with $N$-solitons, we attained some nonlinear localized waves (soliton, breather, lump, and rogue), and their variety of interaction solutions. To the best of the authors' knowledge, all the localized waves and their variety of interaction solutions are entirely new and reported for the first time.

In order to illustrate the physical significance of the dimensionally reduced $(2+1)$ dimensional BqE specified by Eq. (3), we have displayed the 3D plots and their corresponding density views to some of the acquired solutions, which represent the solitons, breathers, lumps, rogue waves, and their interactions. The interactions can occur among one stripe and one breather, one stripe and one lump, two stripes and one breather, one lump and one periodic breather, two stripes and one lump, two breathers, and two lumps. It is mentioned that the 3D plots and their density views are made by Maple software of some of the explored validated solutions with suitable values of the constraint parameters. $N$-soliton solutions of Eq. (3) given by Eq. (14) are presented in Fig. 1. It is apparent from Fig. 1 that the interactions among $N$ soliton solutions are completely elastic. For $N=2$, the cross-stripe, one periodic breather, and one lump soliton are obtained, which are illustrated in Figs. 2-5. For $N=3$, we obtained the interaction solutions among three soliton solutions, viz. two parallel-stripe solitons and one soliton, one stripe and periodic breather, and one stripe and one lump solitons by selecting some special parameters, as shown in Figs. 6- 8, respectively. In addition, for $N=4$, we have also attained the interaction solutions among four soliton solutions, such as two-stripe solitons and one periodic breather, two periodic breathers, one lump and one periodic breather, one lump and two-stripe solitons, and two lump solitons. The mentioned interaction solutions among four solitons are justified through Figs. 9-13. It is pinpoint to mention here that the lump solitons and rogue waves are obtained via the long-wave limit approach imposing some constraint parameters (see Ablowitz and Satsuma [58]). For other solitons, the HBM along with certain conditions on some constraint parameters are employed. Some of the interaction 
solutions of the localized waves are presented and their dynamical features are analyzed through some time evolution plots (see Figs. 6-7 and Figs. 11-13). It is clear clearly from the mentioned time evolution plots that all the wave interactions among solitons are elastic. It is also to be mentioned here that all the reported solutions in this article have diverse structures over the solutions available in the literature, and the choice of the constraint parameters has a great influence on the attained solutions and their propagation behaviors. Tables 1-3 show some mathematical conditions to secure the localized waves and their interaction solutions from the two-soliton, three-soliton, and four-soliton solutions of Eq. (3) on how to select appropriate constraint parameters. It should be pointed out here that no interaction solutions

591 for $N \geq 5$ are explained due to the limitations of our computational scope. However, our future study will concentrate on constructing interaction solutions for the higher values of $N$, and will present how to select some more appropriate constraint parameters for their interaction solutions. The exhibited results reveal that the acquired solitons might be helpful for explaining

595 the wave propagation behavior in shallow water surfaces. The executed approach can be used 596 to determine localized waves and their interaction solutions to any other NLEEs arising in 597 shallow water. 
600 This article does not contain any studies with human or animal subjects.

601

\section{Conflict of interests.}

603

604

605

606

607

608

609

610

611

612

613

614

615

616

617

618

619

620

621

622

623

624

625

626

627

628

629

\section{CRediT authorship contribution statement}

Dipankar Kumar: Conceptualization, Formal analysis, Data curation, Resources, Software, Supervision, Validation, Writing - review editing. Md. Nuruzzaman: Methodology, Formal analysis, Software, Writing - original draft. Gour Chandra Paul (Corresponding author): Data curation, Methodology, Formal analysis, Resources, Supervision, Validation, Writing - review editing. Ashabul Hoque: Formal analysis, Supervision, Validation, Writing - review editing. Finally, all authors have approved this manuscript and take responsibility for the accuracy of its contents.

\section{Data availability statement}

Data sharing not applicable to this article as no datasets were generated or analysed during the current study.

\section{Acknowledgments}

The work is partially supported by a grant from the University Grants Commission, Bangladesh under grant no. 3632104 through the Bangabandhu Sheikh Mujibur Rahman Science and Technology University, Gopalganj, and the first author acknowledges this support. The research is also supported by the National Science and Technology (NST), Government of Bangladesh by providing NST fellowship under grant Number No. 39.00.0000.012.002.04.1906 to the second author and the author acknowledges this support.

\section{References}

1. Huang, L., Yue, Y., Chen, Y.: Localized waves and interaction solutions to a (3+1)-dimensional generalized KP equation. Comput. Math. Appl.76(4). 831-844 (2018) 
2. Li, B. X., Borshch, V., Xiao, R. L., Paladugu, S., Turiv, T., Shiyanovskii, S. V., Lavrentovich, O. D.: Electrically driven three-dimensional solitary waves as director bullets in nematic liquid crystals. Nat. Commun. 9, 2912 (2018)

3. Hirota R. The direct method in soliton theory. 2004, Cambridge University Press, Cambridge. https://doi.org/10.1017/CBO9780511543043

4. Orapine, H. O., Ayankop-Andi, E., Ibeh, G. J.: Analytical and numerical computations of multisolitons in the Korteweg-de Vries (KdV) equation. Appl. Math. 11(07), 511 (2020)

5. Zabusky, N. J., Kruskal, M. D.: Interaction of solitons in a collisionless plasma and recurrence of initial states. Phys. Rev. Lett. 15, 240-243 (1965)

6. Aya, S., Araoka. F.: Kinetics of motile solitons in nematic liquid crystals. Nat. Commun. 11, 3248 (2020). https://doi.org/10.1038/s41467-020-16864-8

7. Liu, W., Liu, Y., Zhang, Y., Shi, D.: Riemann-Hilbert approach for multi-soliton solutions of a fourth-order nonlinear Schrödinger equation. Mod. Phys. Letts. B. 33(33), 1950416 (2019)

8. Chabchoub, A., Hoffmann, N.P., Akhmediev, N.: Rogue wave observation in a water wave tank. Phys. Rev. Lett. 106(20), 204502 (2011)

9. Paul, G. C., Eti, F. Z., Kumar, D.: Dynamical analysis of lump, lump-triangular periodic, predictable rogue and breather wave solutions to the (3+1)-dimensional gKP-Boussinesq equation. Results in Physics. 19, 103525 (2020)

10. Wu, J., Liu, Y., Piao, L., Zhuang, J., Wang, D. S.: Nonlinear localized waves resonance and interaction solutions of the (3+1)-dimensional Boiti-Leon-Manna-Pempinelli equation. Nonlinear Dyn. 100, 1527-1541 (2020)

11. Dudley, J.M., Genty, G., Mussot, A., Chabchoub, A., Dias, F.: Rogue waves and analogies in optics and oceanography. Nat. Rev. Phys. 1(11), 675-689 (2019)

12. Yu, W., Zhang, H., Zhou, Q., Biswas, A., Alzahrani, A. K., Liu, W.: The mixed interaction of localized, breather, exploding and solitary wave for the $(3+1)$-dimensional KadomtsevPetviashvili equation in fluid dynamics. Nonlinear Dyn. 100(2), 1611-1619 (2020)

13. Nestor, S., Abbagari, S., Houwe, A., Betchewe, G., Doka, S. Y.: Diverse chirped optical solitons and new complex traveling waves in nonlinear optical fibers. Commun. Theor. Phys. 72(6), $065501(2020)$

14. Xu, T., Chen, Y., Lin, J.: Localized waves of the coupled cubic-quintic nonlinear Schrödinger equations in nonlinear optics. Chin. Phys. B. 26(12), 120201 (2017)

15. Liu, Y., Wen, X. Y., Wang, D. S.: Novel interaction phenomena of localized waves in the generalized (3+1)-dimensional KP equation. Comput. Math. Appl. 78(1), 1-9 (2019)

16. Li, Z. Q., Tian, S. F., Peng, W. Q., Yang, J. J.: Inverse Scattering Transform and Soliton Classification of Higher-Order Nonlinear Schrödinger-Maxwell-Bloch Equations. Theor. Math. Phys. 203(3), 709-725 (2020) 
17. Lü, X., Hua, Y. F., Chen, S. J., Tang, X. F.: Integrability characteristics of a novel (2+1)dimensional nonlinear model: Painlevé analysis, soliton solutions, Bäcklund transformation, Lax pair and infinitely many conservation laws. Commun. Nonlinear Sci. Numer. Simul. 95, 105612 (2021)

18. Yang, Y., Suzuki, T., Cheng, X.: Darboux transformations and exact solutions for the integrable nonlocal Lakshmanan-Porsezian-Daniel equation. Appl. Math. Letts. 99, 105998 (2020)

19. Ryabov, P. N., Sinelshchikov, D. I., Kochanov, M. B.: Application of the Kudryashov method for finding exact solutions of the high order nonlinear evolution equations. Appl. Math. Comput. 218(7), 39653972 (2011)

20. Kumar, D., Seadawy, A. R., Joardar, A. K.: Modified Kudryashov method via new exact solutions for some conformable fractional differential equations arising in mathematical biology. Chin. J. Phys. 56(1), 75-85 (2018)

21. Kumar, D., Kaplan, M.: Application of the modified Kudryashov method to the generalized Schrödinger-Boussinesq equations. Opt. Quant. Electron. 50(9), 1-14 (2018)

22. Kumar, D., Paul, G. C., Biswas, T., Seadawy, A. R., Baowali, R., Kamal, M., Rezazadeh, H.: Optical solutions to the Kundu-Mukherjee-Naskar equation: mathematical and graphical analysis with oblique wave propagation. Phys. Scr. 96(2), 025218 (2020)

23. Ahmed, H. M., Rabie, W. B., Ragusa, M. A.: Optical solitons and other solutions to Kaup-Newell equation with Jacobi elliptic function expansion method. Anal. Math. Phys. 11(1), 1-6 (2021)

24. Kumar, D., Hosseini, K., Samadani, F.: The sine-Gordon expansion method to look for the traveling wave solutions of the Tzitzéica type equations in nonlinear optics. Optik. 149, 439-446 (2017)

25. Kumar, D., Manafian, J., Hawlader, F., Ranjbaran, A.: New closed form soliton and other solutions of the Kundu-Eckhaus equation via the extended sinh-Gordon equation expansion method. Optik. 160, 159-167 (2018)

26. Seadawy, A. R., Kumar, D., Chakrabarty, A. K.: Dispersive optical soliton solutions for the hyperbolic and cubic-quintic nonlinear Schrödinger equations via the extended sinh-Gordon equation expansion method. Eur. Phys. J. Plus. 133(5), 182 (2018)

27. Kumar, D., Joardar, A. K., Hoque, A., Paul, G. C.: Investigation of dynamics of nematicons in liquid crystals by extended sinh-Gordon equation expansion method. Opt. Quant. Electron. 51(7), 1-36 (2019)

28. Kumar, D., Paul, G. C.: Solitary and periodic wave solutions to the family of nonlinear conformable fractional Boussinesq-like equations. Math. Meth. Appl Sci. 44(4), 3138-3158 (2021)

29. Kumar, D., Paul, G. C., Mondal, J., Islam, A. S.: On the propagation of alphabetic-shaped solitons to the (2+1)-dimensional fractional electrical transmission line model with wave obliqueness. Res. Phys. 19, 103641 (2020) 
30. Kumar, D., Park, C., Tamanna, N., Paul, G. C., Osman, M. S.: Dynamics of two-mode SawadaKotera equation: Mathematical and graphical analysis of its dual-wave solutions. Res. Phys. 19, $103581(2020)$

31. Cui, C.J., Tang, X.Y., Cui, Y.J.: New variable separation solutions and wave interactions for the (3+1)-dimensional Boiti-Leon-Manna-Pempinelli equation. Appl. Math. Letts. 102, 106109 (2020)

32. Wang, D. S., Guo, B., Wang, X.: Long-time asymptotics of the focusing Kundu-Eckhaus equation with nonzero boundary conditions. J. Differ. Equ. 266(9), 5209-5253 (2019)

33. Kaur, L., Wazwaz, A.M.: Painlevé analysis and invariant solutions of generalized fifth-order nonlinear integrable equation. Nonlinear Dyn. 94(4), 2469-2477 (2018)

34. Ren, B., Lin, J., Lou, Z. M.: Consistent Riccati expansion and rational solutions of the Drinfel'dSokolov-Wilson equation. Appl. Math. Letts. 105, 106326 (2020)

35. Dong, M.J., Tian, S.F., Yan, X.W., Zou, L.: Solitary waves, homoclinic breather waves and rogue waves of the (3+1)-dimensional Hirota bilinear equation. Comput. Math. Appl. 75(3), 957-964 (2018)

36. Hua, Y. F., Guo, B. L., Ma, W. X., Lü, X.: Interaction behavior associated with a generalized $(2+1)$-dimensional Hirota bilinear equation for nonlinear waves. Appl. Math. Modell. 74, 184198 (2019)

37. He, B., Meng, Q.: Lump and interaction solutions for a generalized (3+1)-dimensional propagation model of nonlinear waves in fluid dynamics. Int. J. Comput. Math. 98(3), 592-607 (2021)

38. Kumar, D., Kuo, C. K., Paul, G. C., Saha, J., Jahan, I.: Wave propagation of resonance multistripes, complexitons, and lump and its variety interaction solutions to the (2+1)-dimensional pKP equation. Commun. Nonlinear Sci. Numer. Simul. 100, 105853 (2021)

39. Yue, Y., Huang, L., Chen, Y.: Localized waves and interaction solutions to an extended (3+1)dimensional Jimbo-Miwa equation. Appl. Math. Letts. 89, 70-77 (2019)

40. Rao, J., He, J., Mihalache, D., Cheng, Y.: Dynamics and interaction scenarios of localized wave structures in the Kadomtsev-Petviashvili-based system. Appl. Math. Letts. 94, 166-173 (2019)

41. Liu, Y., Wen, X. Y., Wang, D. S.: The N-soliton solution and localized wave interaction solutions of the (2+1)-dimensional generalized Hirota-Satsuma-Ito equation. Comput. Math. Appl. 77(4), 947-966 (2019)

42. Zhang, W. J, Xia, T. C.: Solitary wave, M-lump and localized interaction solutions to the (4+1)dimensional Fokas equation. Phys. Scr. 95(4), 045217 (2020).

43. Sun, L., Qi, J., An, H.: Novel localized wave solutions of the (2+1)-dimensional Boiti-LeonManna-Pempinelli equation. Commun. Theor. Phys. 72(12), 125009 (2020)

44. Song, N., Xue, H., Xue, Y. K.: Dynamics of higher-order localized waves for a coupled nonlinear Schrödinger equation. Commun. Nonlinear Sci. Numer. Simul. 82, 105046 (2020) 
45. Ma, Y. L.: N-solitons, breathers and rogue waves for a generalized Boussinesq equation. Int. J. Comp. Math. 97(8), 1648-1661 (2020)

46. Vinodh, D., Asokan, R.: Multi-soliton, rogue wave and periodic wave solutions of generalized (2+1)- dimensional Boussinesq equation. Int. J. Appl. Comput. Math. 6(1), 1-6 (2020)

47. Liu, W., Zhang, Y.: Dynamics of localized waves and interaction solutions for the $(3+1)$ dimensional B-type Kadomtsev-Petviashvili-Boussinesq equation. Adv. Differ. Equ. 2020(1), $1-12(2020)$

48. Yue, Y., Chen, Y.: Dynamics of localized waves in a (3+1)-dimensional nonlinear evolution equation. Mod. Phys. Letts. B. 33(09), 1950101 (2019)

49. Wazwaz, A. M.: New travelling wave solutions to the Boussinesq and the Klein-Gordon equations. Commun. Nonlinear Sci. Numer. Simul. 13(5), 889-901 (2008)

50. Jawad, A.M., Petković, M.D., Laketa, P., Biswas, A.: Dynamics of shallow water waves with Boussinesq equation. Scientia Iranica. 20(1), 179-184 (2013)

51. Lin, Q., Wu, Y. H., Loxton, R., Lai, S.: Linear B-spline finite element method for the improved Boussinesq equation. J. Comput. Appl. Math. 224(2), 658-667 (2019)

52. Wazwaz, A. M., Kaur, L.: New integrable Boussinesq equations of distinct dimensions with diverse variety of soliton solutions. Nonlinear Dyn. 97(1), 83-94 (2019)

53. Zou, H., Li, H., Liu, X., Liu, A.: The application of a numerical model to coastal surface water waves. J Ocean Univ. China. 4(2), 177-184 (2005)

54. Droenen, N., Deigaard, R.: Adaptation of a Boussinesq wave model for dune erosion modeling. Coastal Eng. Proceedings. 33, 31-31 (2012)

55. Kirby, J, T.: Boussinesq models and their application to coastal processes across a wide range of scales. J. Waterw. Port Coast. Ocean Engrg. 142(6), (2016)

56. Lynett, P. J., Melby, J. A., Kim, D. H.: An application of Boussinesq modeling to hurricane wave overtopping and inundation. Ocean Eng. 37(1), 135-153 (2010)

57. Roeber, V., Cheung, K. F., Kobayashi, M. H.: Shock-capturing Boussinesq-type model for nearshore wave processes. Coastal Eng. 57(4), 407-423 (2010)

58. Ablowitz, M.J., Satsuma, J.: Solitons and rational solutions of nonlinear evolution equations. J. Math. Phys. 19(10), 2180-2186 (1978) 


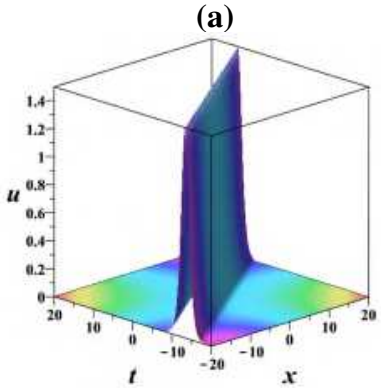

(e)

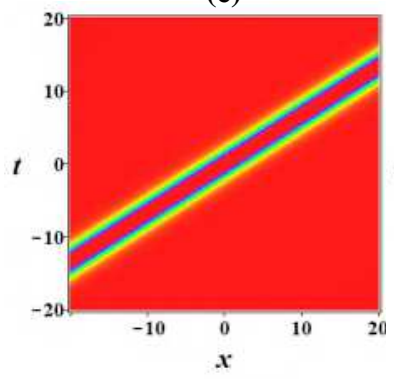

(b)

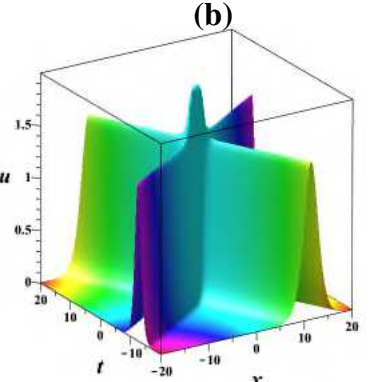

(f)

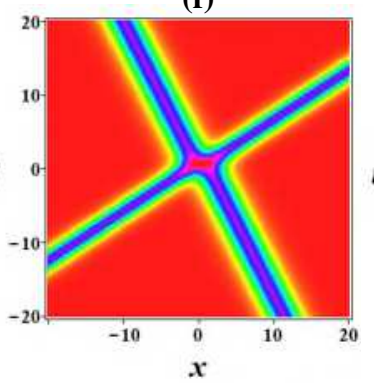

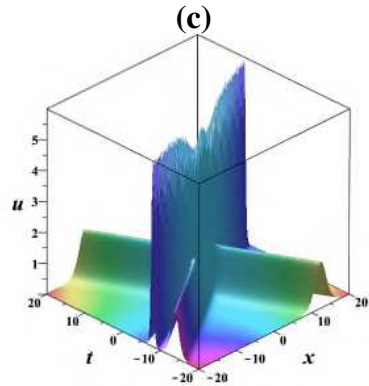

(g)

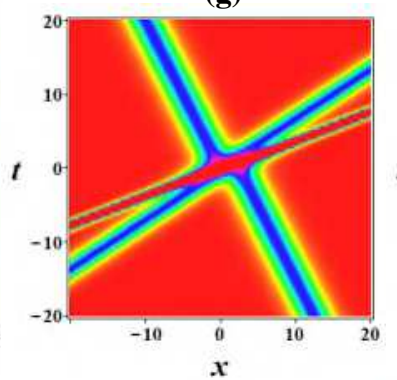

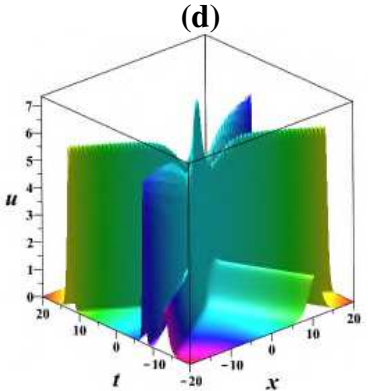

(h)

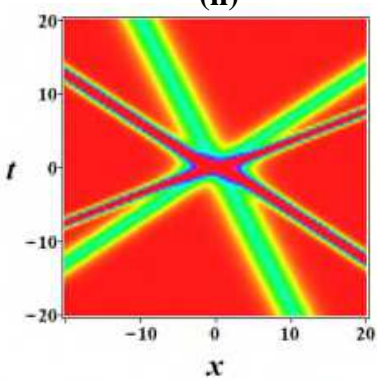

Figure 1. Graphical illustration of $N$-soliton solutions given by Eq. (14) of Eq. (3) with $a_{1}=$ $1, a_{2}=-1, a_{3}=2, a_{4}=-2, b_{1}=1, b_{2}=-1, b_{3}=2, b_{4}=-2, \omega_{1}=0, \omega_{2}=0, \omega_{3}=$ $0, \omega_{4}=0, \alpha=1, \beta=1, \gamma=1, \delta=1$, and $y=0$. The 3D plots for (a) $N=1$, (b) $N=2$, (c) $N=3$, and (d) $N=4$. (e)-(h) The density views of (a)-(d), respectively.

(a)

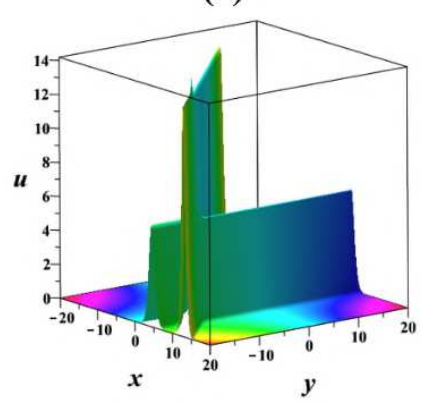

(d)

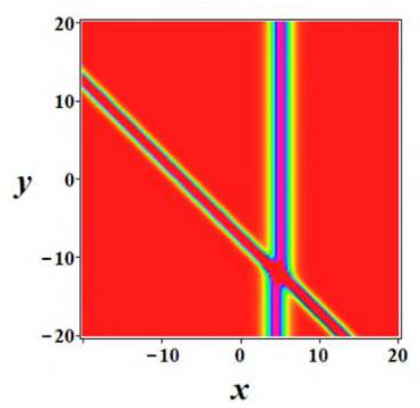

(b)

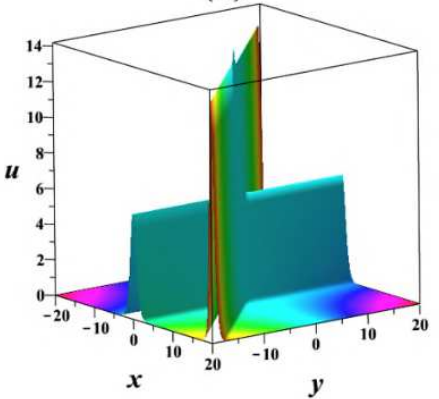

(e)

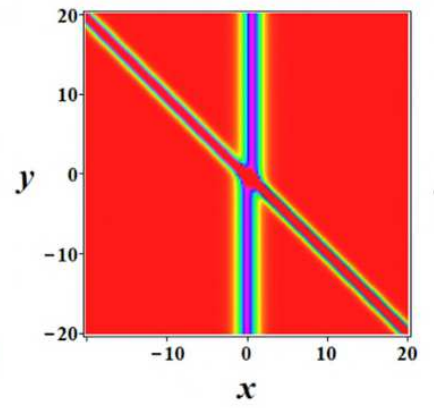

(c)

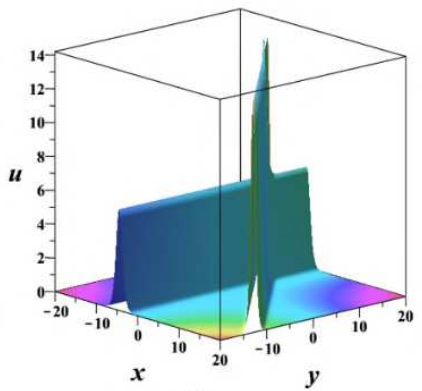

(f)

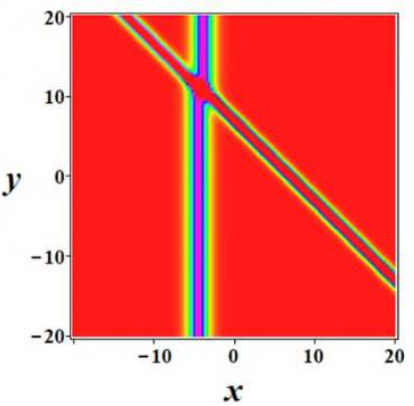

Figure 2. Time evolution 3D plots of two cross-stripe soliton solutions given by Eq. (18) at (a) $t=-5$, (b) $t=0$, and (c) $t=5$ with $p_{1}=3, p_{2}=-2, q_{1}=3, q_{2}=0, \omega_{1}=0, \omega_{2}=0$, $\alpha=1, \beta=1, \gamma=1, \delta=1$. (d)-(f) The density views of (a)-(c), respectively. 
(a)

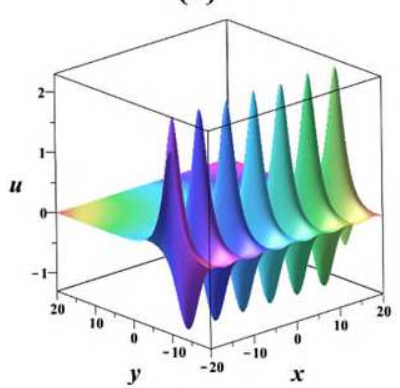

(d)

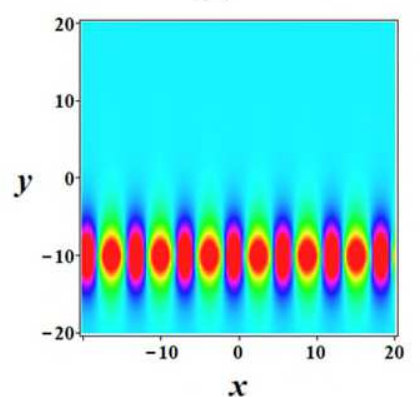

(b)

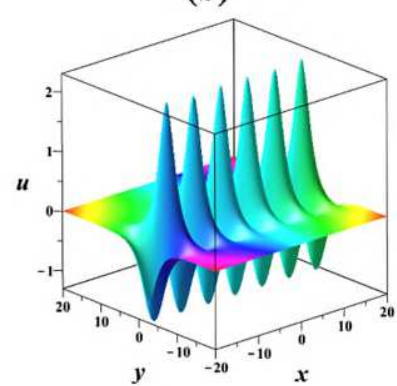

(e)

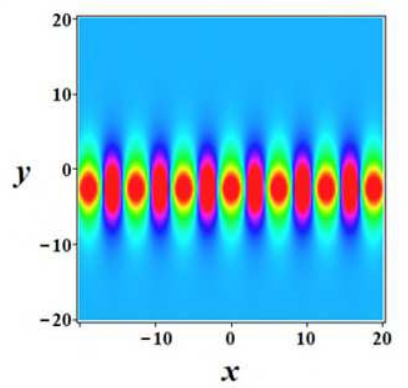

(c)

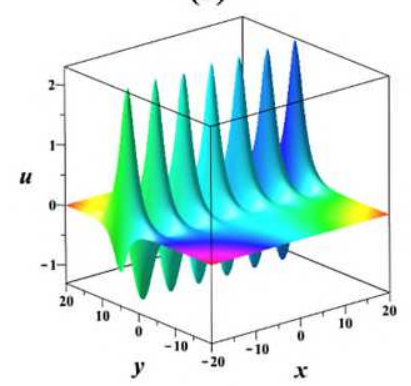

(f)

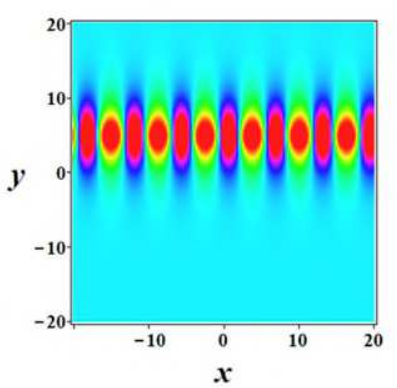

Figure 3. Time evolution 3D plots of one $x$-periodic breather wave solutions given by Eq. (21) 783 at (a) $t=-5$, (b) $t=0$, and (c) $t=5$ with $a_{1}=a_{2}^{*}=I, b_{1}=0.5, b_{2}=0.5, \omega_{1}=0, \omega_{2}=$ 784 $0, \alpha=1, \beta=1, \gamma=1, \delta=1$. (d)-(f) The density views of (a)-(c), respectively.

785

(a)

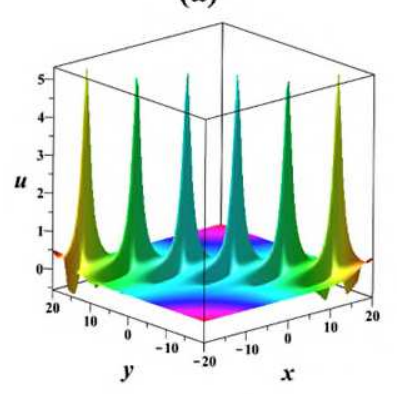

(b)

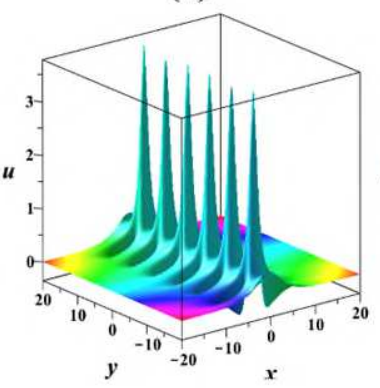

(c)

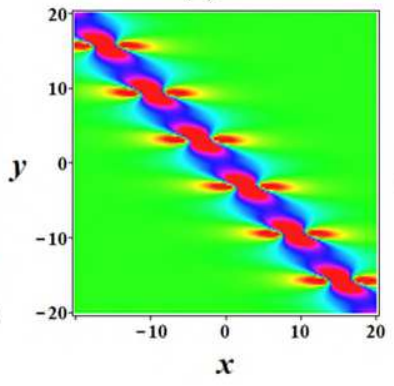

(d)

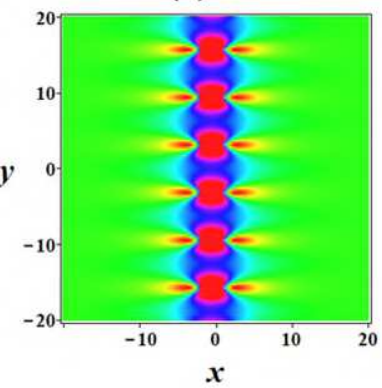

787 Figure 4. Periodic breather solutions given by Eq. (23) at $t=0$. The 3D plots of (a) one $x y$ 788 periodic breather with $a_{1}=a_{2}=\frac{1}{3}, b_{1}=b_{2}^{*}=\frac{1}{3}+I, \omega_{1}=\omega_{2}=0, \alpha=1, \beta=1, \gamma=1$, 789 $\delta=1$, and (b) one $y$-periodic breather with $a_{1}=a_{2}=\frac{1}{3}, b_{1}=b_{2}^{*}=I, \omega_{1}=\omega_{2}=0, \alpha=1$, 790 $\beta=1, \gamma=1, \delta=1$. (c)-(d) The density views of (a)-(b), respectively. 
(a)

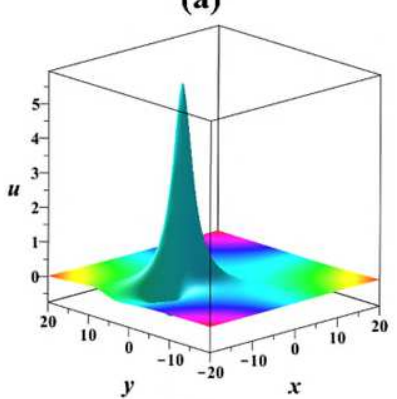

(d)

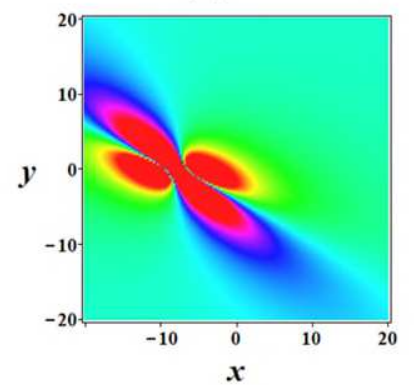

(b)

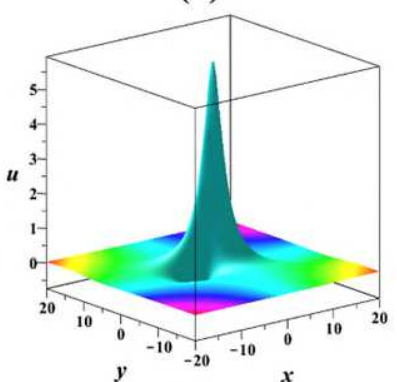

(e)

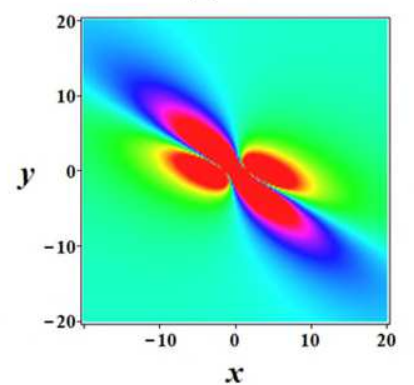

(c)

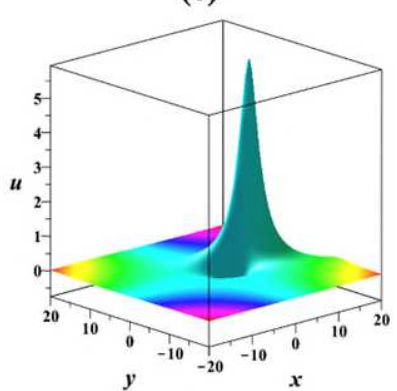

(f)

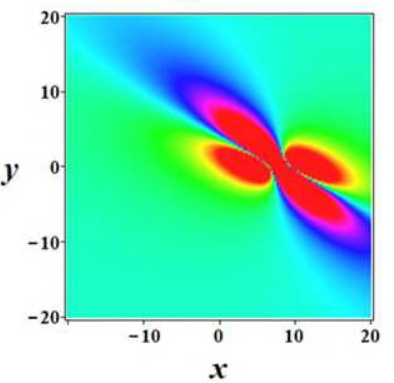

Figure 5. Time evolution 3D plots of lump soliton solutions given by Eq. (25) at (a) $t=-5$, 793 (b) $t=0$, and (c) $t=5$ under the specified conditions $m_{1}=m_{2}^{*}=1+I, \alpha=1, \beta=1, \gamma=$ $7941, \delta=1$. (d)-(f) The density views of (a)-(c), respectively.

(a)

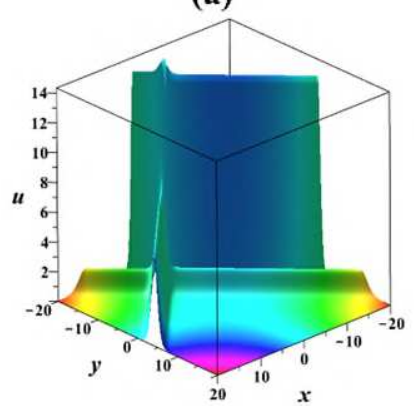

(d)

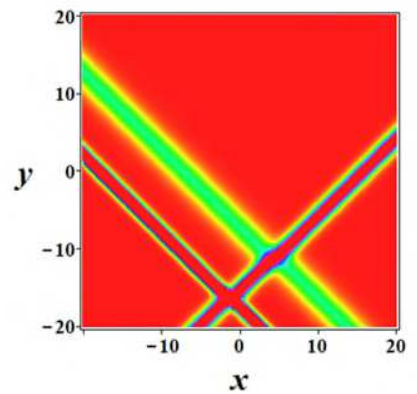

(b)

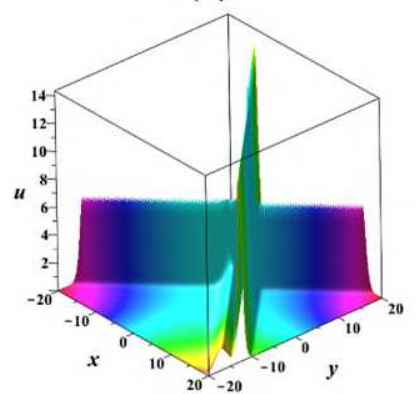

(e)

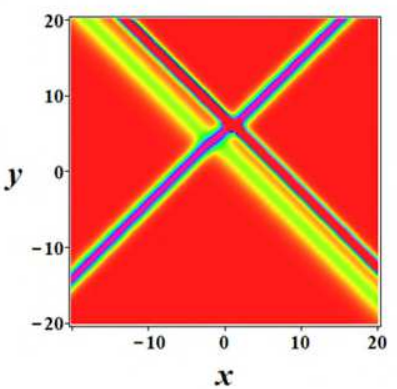

(c)

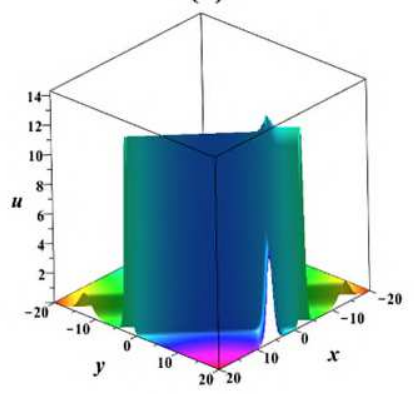

(f)

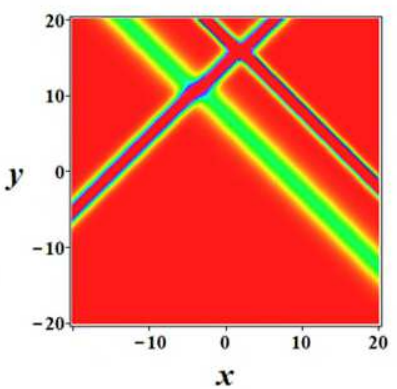

Figure 6. Time evolution 3D plots of the interaction of two parallel-stripe solitons with another soliton given by Eq. (29) at (a) $t=-5$, (b) $t=2$, and (c) $t=5$ under suitable selection of constraint parameters as $p_{1}=3, p_{2}=-2, p_{3}=1, q_{1}=3, q_{2}=2, q_{3}=1, \omega_{1}=0, \omega_{2}=$ $0, \omega_{3}=0, \alpha=1, \beta=1, \gamma=1, \delta=1$. (d)-(f) The density views of (a)-(c), respectively. 
(a)

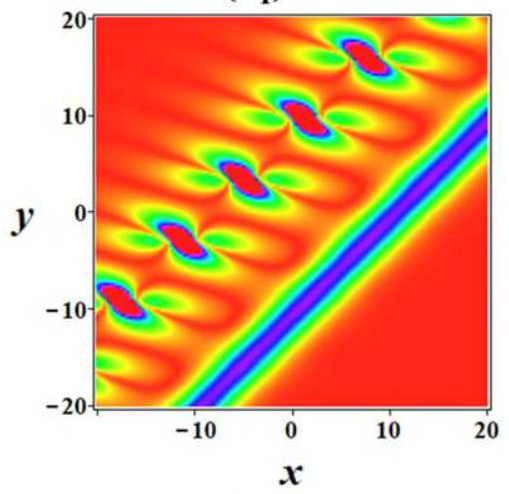

(b)

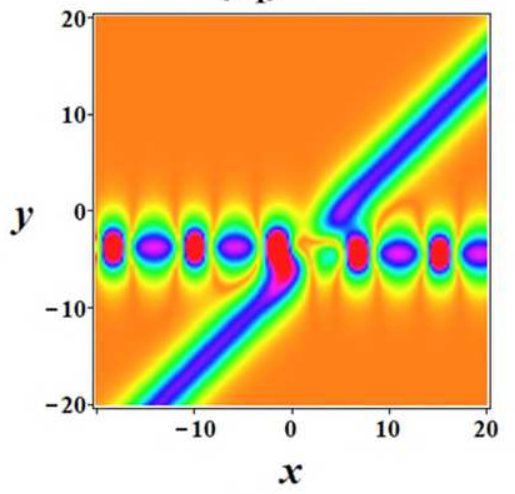

(c)

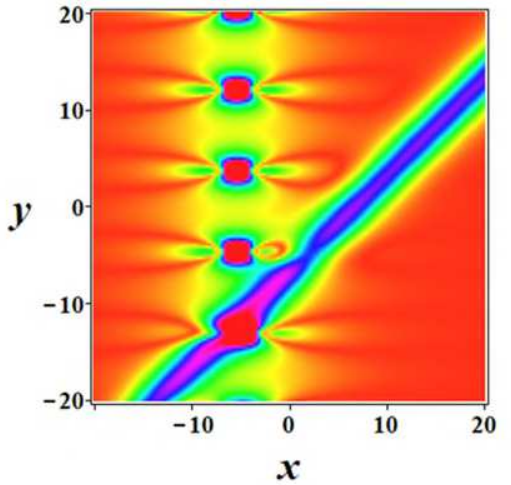

$\left(a_{2}\right)$

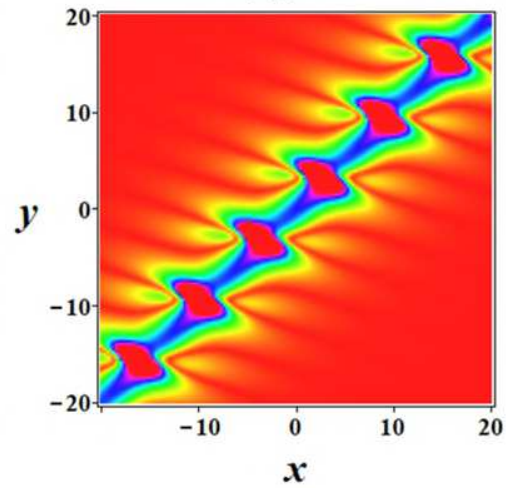

$\left(b_{2}\right)$

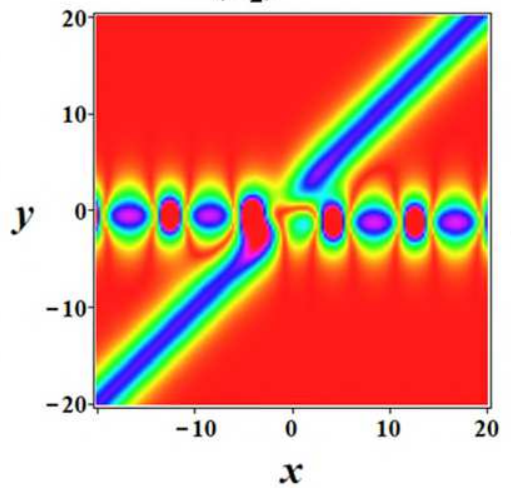

(c $\left.c_{2}\right)$

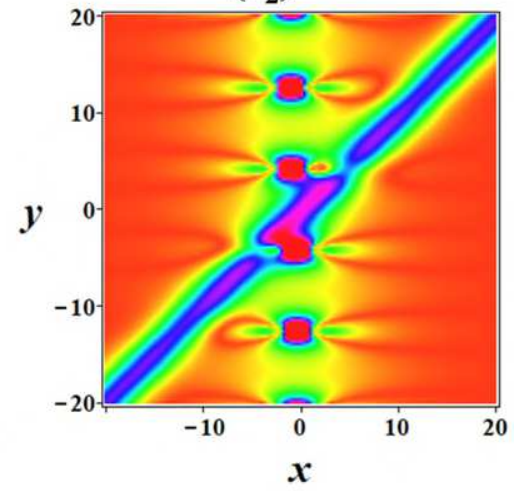

$\left(a_{3}\right)$

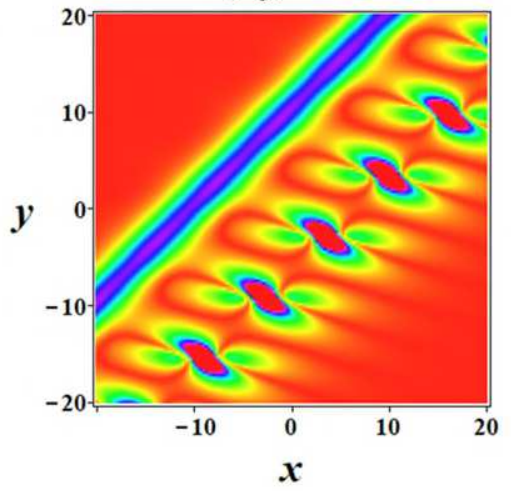

(b)

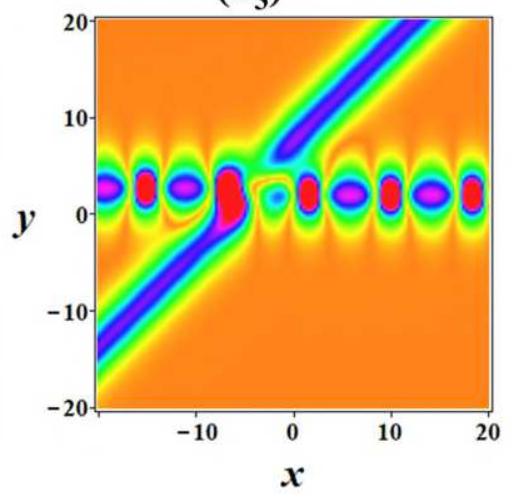

(c $\left.c_{3}\right)$

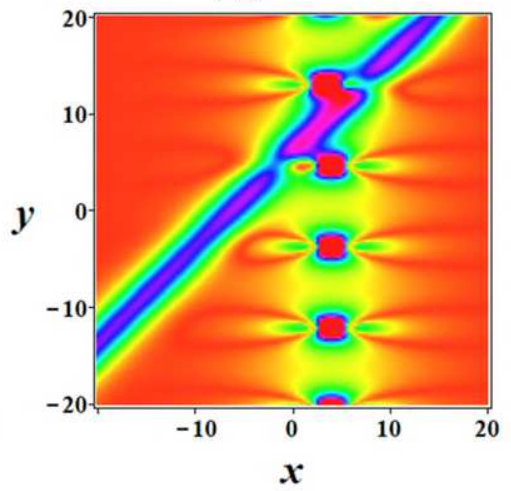

Figure 7. Time evolution density plots of the interaction solutions between one-stripe soliton and one periodic breather given by Eq. (29) at $\left(\mathrm{a}_{1}\right)-\left(\mathrm{c}_{1}\right) t=-5,\left(\mathrm{a}_{2}\right)-\left(\mathrm{c}_{2}\right) t=0$, and $\left(\mathrm{a}_{3}\right)-\left(\mathrm{c}_{3}\right)$ $t=5$. ( (a 1$)-\left(\mathrm{a}_{3}\right)$ One stripe soliton and one $x y$-periodic breather with $a_{1}=a_{2}=0.25, a_{3}=$ $-0.75, b_{1}=b_{2}^{*}=-0.25+0.75 I, b_{3}=0.75, \omega_{1}=0, \omega_{2}=0, \omega_{3}=0, \alpha=1, \beta=1, \gamma=$ $1, \delta=1,\left(\mathrm{~b}_{1}\right)-\left(\mathrm{b}_{3}\right)$ One stripe soliton and one $x$-periodic breather with $a_{1}=a_{2}^{*}=0.25 I, a_{3}=$ $-0.75, b_{1}=b_{2}=-0.25, b_{3}=0.75, \omega_{1}=0, \omega_{2}=0, \omega_{3}=0, \alpha=1, \beta=1, \gamma=1, \delta=1$, and $\left(\mathrm{c}_{1}\right)-\left(\mathrm{c}_{3}\right)$ One stripe soliton and one $y$-periodic breather with $a_{1}=a_{2}=0.25, a_{3}=-0.75$, $b_{1}=b_{2}^{*}=0.75 I, b_{3}=0.75, \omega_{1}=0, \omega_{2}=0, \omega_{3}=0, \alpha=1, \beta=1, \gamma=1, \delta=1$. 


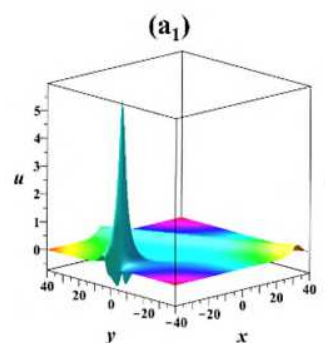

(b)

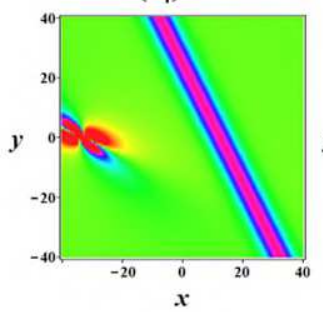

$\left(a_{2}\right)$

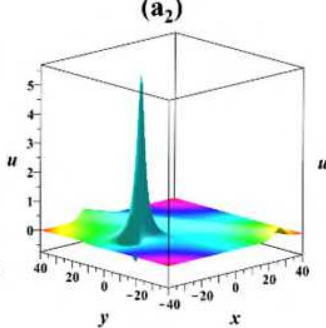

(b)

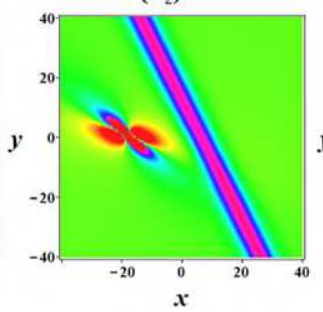

$\left(a_{3}\right)$

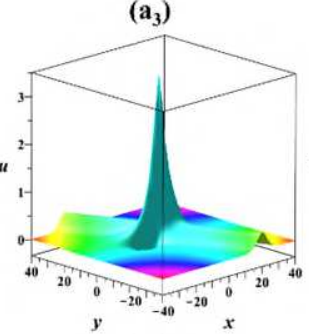

(b)

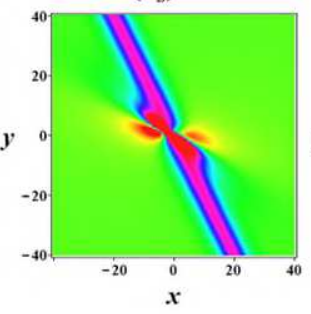

$\left(a_{4}\right)$

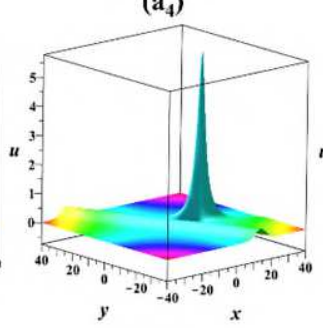

(b)

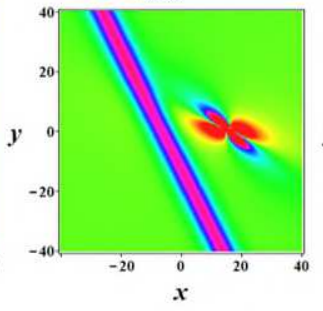

(a)

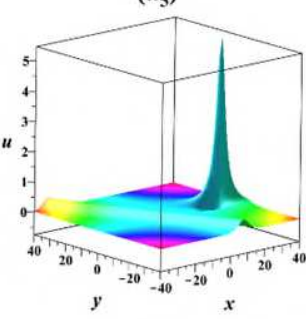

(b)

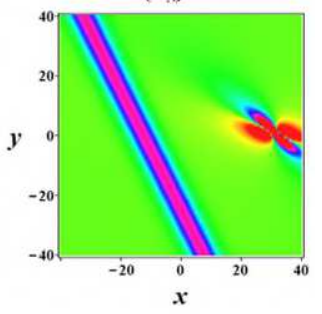

810

811

812

813

Figure 8. Time evolution 3D plots of lump-stripe solitons given by Eq. (34) at $\left(\mathrm{a}_{1}\right) t=-20$, (a $) t=-10,\left(\mathrm{a}_{3}\right) t=0,\left(\mathrm{a}_{4}\right) t=10$, and (as) $t=20$ with $m_{1}=(1+I), m_{2}=(1-I)$, $m_{3}=0.5, a_{3}=-0.5, \alpha=1, \beta=1, \gamma=1, \delta=1$. ( $\left.b_{1}\right)-\left(b_{5}\right)$ The density views of (a 1 )-(a5), respectively.

(a)

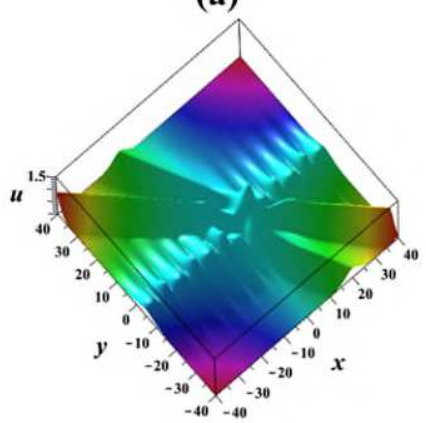

(d)

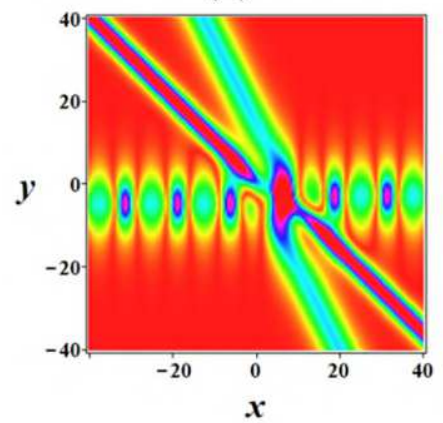

(b)

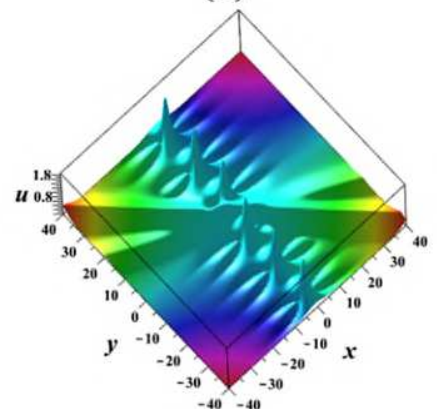

(e)

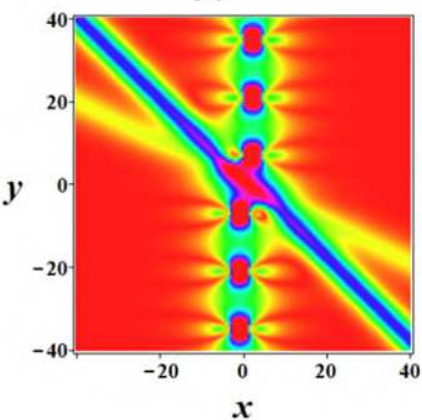

(c)

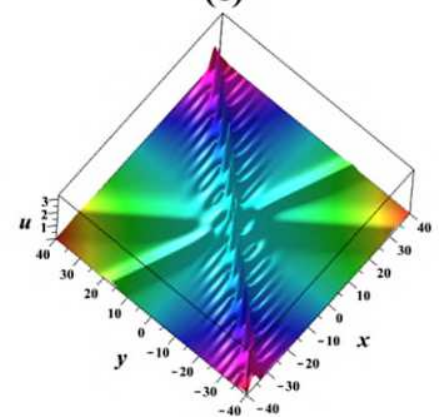

(f)

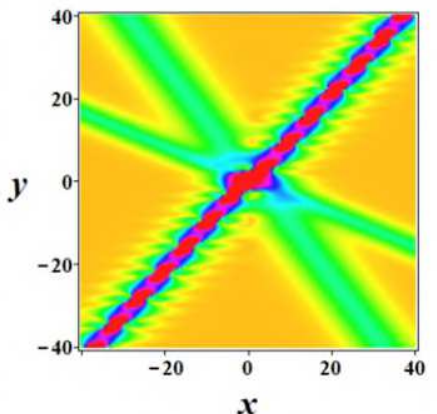

Figure 9. Interaction solutions among two-stripe solitons and one periodic breather given by Eq. (40) at $t=0$. The 3D plots of (a) two-stripe solitons and one $x$-periodic breather with $a_{1}=$ $a_{2}^{*}=0.50 I, a_{3}=0.50, a_{4}=0.75, b_{1}=b_{2}=0.25, b_{3}=0.25, b_{4}=0.75, \omega_{1}=0, \omega_{2}=0$, $\omega_{3}=0, \omega_{4}=0, \alpha=1, \beta=1, \gamma=1, \delta=1$, (b) two-stripe solitons and one $y$-periodic breather with $a_{1}=a_{2}=0.25, a_{3}=0.25, a_{4}=0.50, b_{1}=b_{2}^{*}=0.45 I, b_{3}=0.50, b_{4}=$ $0.50, \omega_{1}=0, \omega_{2}=0, \omega_{3}=0, \omega_{4}=0, \alpha=1, \beta=1, \gamma=1, \delta=1$, and (c) two-stripe solitons and one $x y$-periodic breather with $a_{1}=a_{2}=\frac{1}{3}, a_{3}=\frac{1}{3}, a_{4}=\frac{1}{3}, b_{1}=b_{2}^{*}=-0.3+I$, $b_{3}=0.8, b_{4}=0.25, \omega_{1}=0, \omega_{2}=0, \omega_{3}=0, \omega_{4}=0, \alpha=1, \beta=1, \gamma=1, \delta=1$. (d)-(f) The density views of (a)-(c), respectively. 
(a)

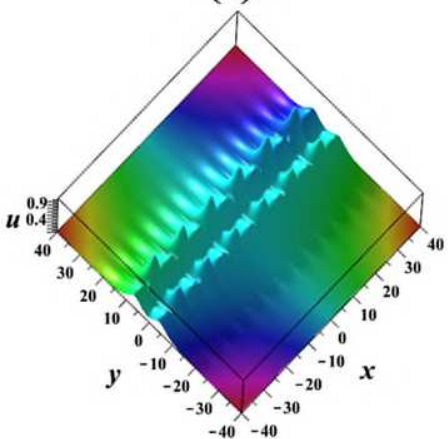

(d)

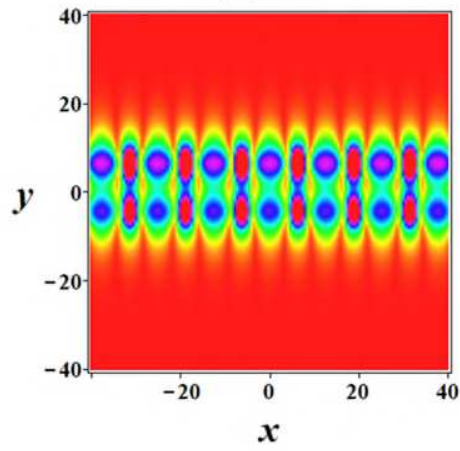

(b)

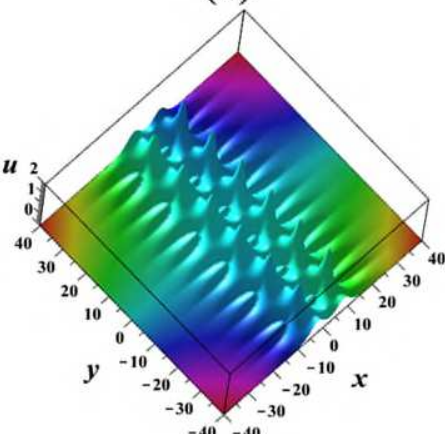

(e)

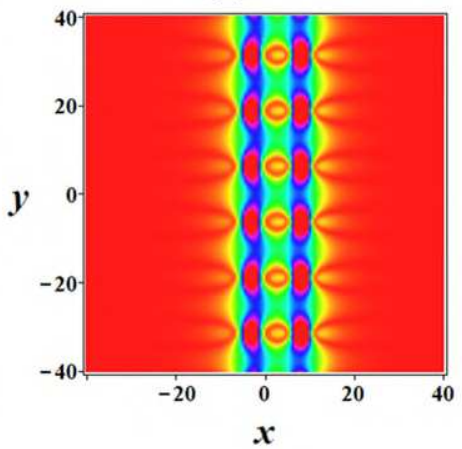

(c)

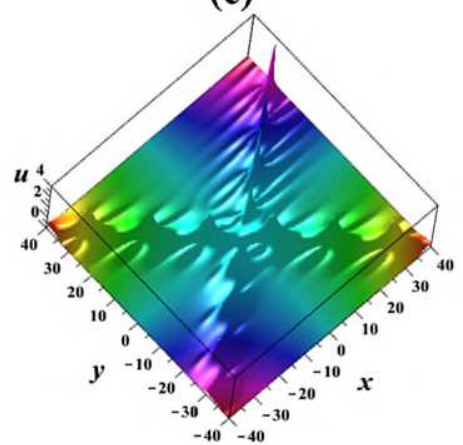

(f)

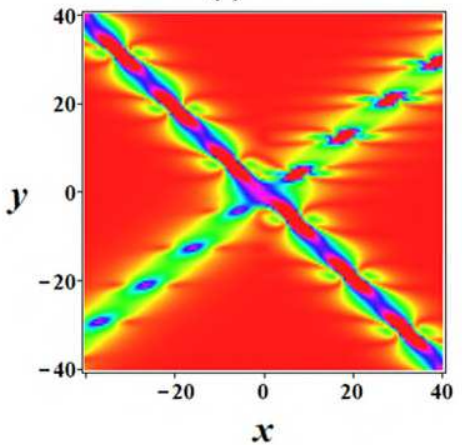

Figure 10. Interaction solutions between two periodic breathers given by Eq. (40) at $t=0$. The 3D plots of (a) two parallel $x$-periodic breathers with $a_{1}=a_{2}^{*}=0.50 I, a_{3}=a_{4}^{*}=0.50 I$, $b_{1}=b_{2}=0.3, b_{3}=b_{4}=0.50, \omega_{1}=0, \omega_{2}=0, \omega_{3}=0, \omega_{4}=0, \alpha=1, \beta=1, \gamma=1$, $\delta=1$, (b) two parallel $y$-periodic breathers with $a_{1}=a_{2}=0.25, a_{3}=a_{4}=0.50, b_{1}=b_{2}^{*}=$ $-0.50 I, b_{3}=b_{4}^{*}=0.50 I, \omega_{1}=0, \omega_{2}=0, \omega_{3}=0, \omega_{4}=0, \alpha=1, \beta=1, \gamma=1, \delta=1$, and (c) two cross $x y$-periodic breathers with $a_{1}=a_{2}=0.35, a_{3}=a_{4}=0.2, b_{1}=b_{2}^{*}=$ $0.35-0.50 I, b_{3}=b_{4}^{*}=-0.25+0.75 I, \omega_{1}=0, \omega_{2}=0, \omega_{3}=0, \omega_{4}=0, \alpha=1, \beta=1$, $\gamma=1, \delta=1$. (d)-(f) The density views of (a)-(c), respectively.

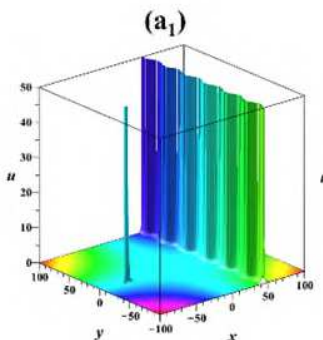

$\left(b_{1}\right)$

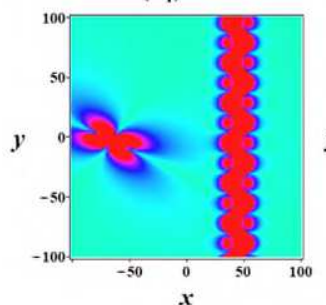

$\left(a_{2}\right)$

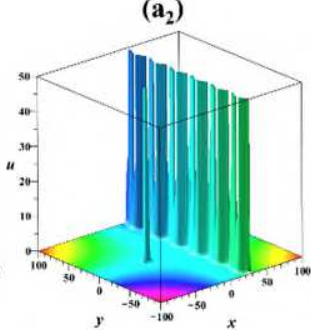

$\left(b_{2}\right)$

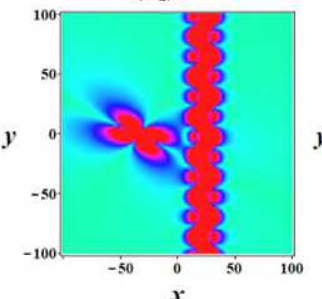

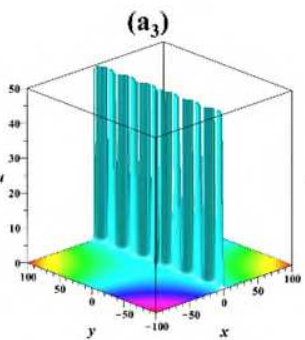

$\left(\mathbf{b}_{3}\right)$

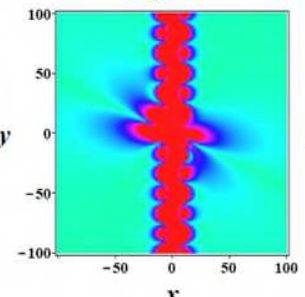

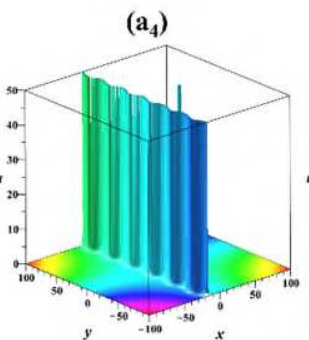

$\left(\mathbf{b}_{4}\right)$

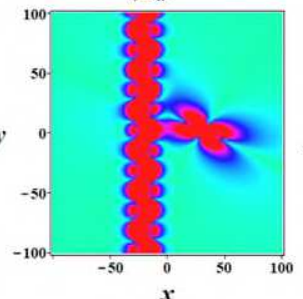

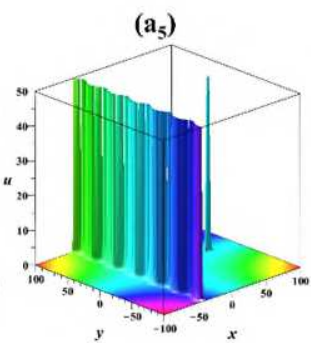

$\left(\mathbf{b}_{5}\right)$

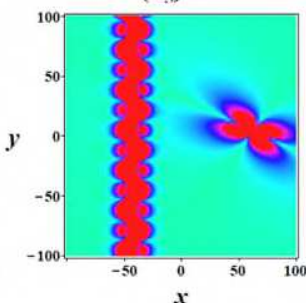

Figure 11. Time evolution $3 \mathrm{D}$ plots of the interaction solutions between one lump and one periodic breather given by Eq. (43) at $\left(\mathrm{a}_{1}\right) t=-40,\left(\mathrm{a}_{2}\right) t=-20,\left(\mathrm{a}_{3}\right) t=0,\left(\mathrm{a}_{4}\right) t=20$, and (as) $t=40$ under constraint parameters as $m_{1}=m_{2}^{*}=(0.5+1.5 I), \quad m_{3}=m_{4}^{*}=$ $0.75 I, a_{3}=-0.25, a_{4}=-0.25, \alpha=1, \beta=1, \gamma=1, \delta=1$. (b $\left.b_{1}\right)-\left(b_{5}\right)$ The density views of (a) $-\left(a_{5}\right)$, respectively. 


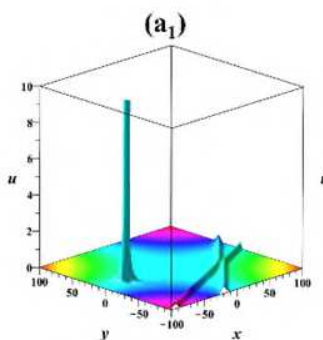

(b)

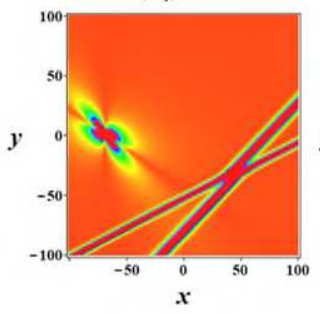

$\left(a_{2}\right)$

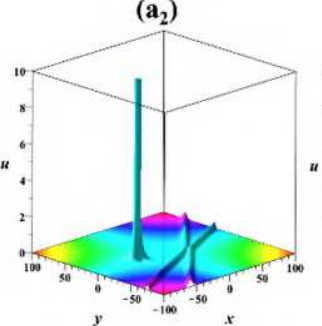

(b)

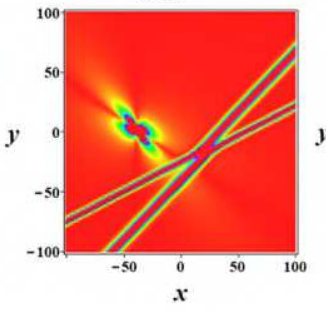

$\left(a_{3}\right)$

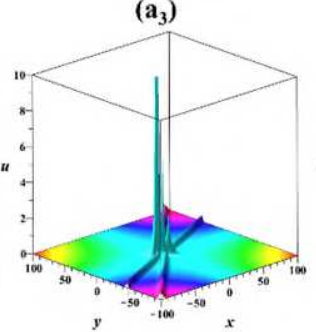

(b)

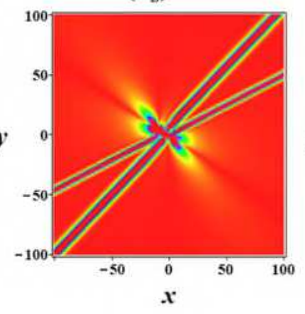

$\left(a_{4}\right)$

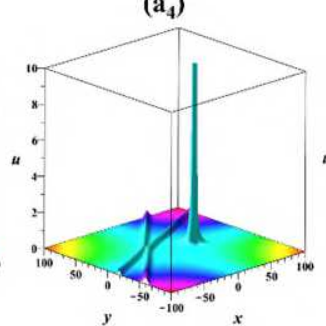

(b)

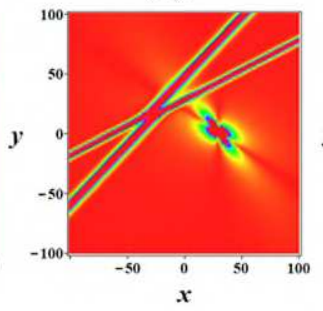

(a)

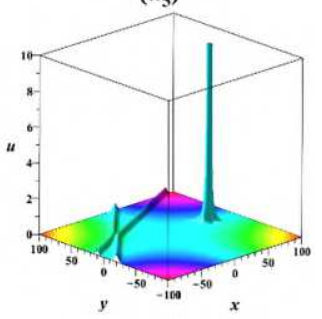

(b)

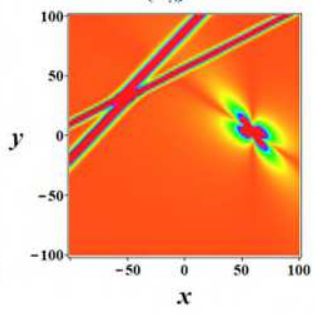

Figure 12. Time evolution 3D plots of the interaction solutions between one lump and two-

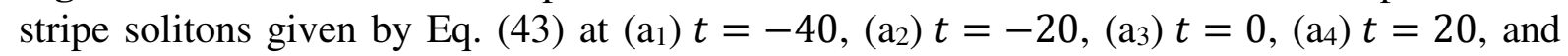
(a) $t=40$ with $m_{1}=m_{2}^{*}=(0.75+0.75 I), m_{3}=-2, m_{4}=-1, a_{3}=-0.6, a_{4}=-0.6$, $\alpha=1, \beta=1, \gamma=1, \delta=1$. ( $\left.\mathrm{b}_{1}\right)-\left(\mathrm{b}_{5}\right)$ The density views of $\left(\mathrm{a}_{1}\right)-\left(\mathrm{a}_{5}\right)$, respectively.

(a)

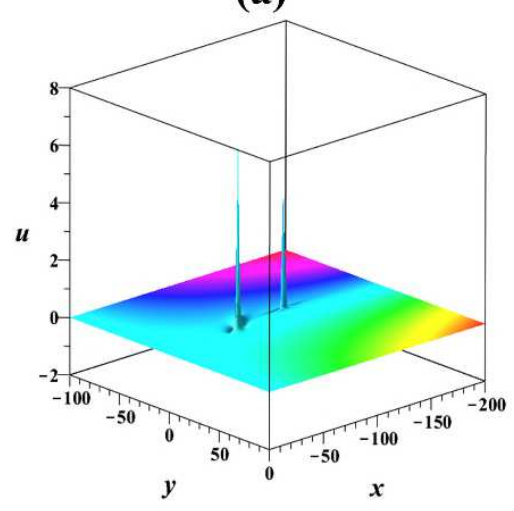

(d)
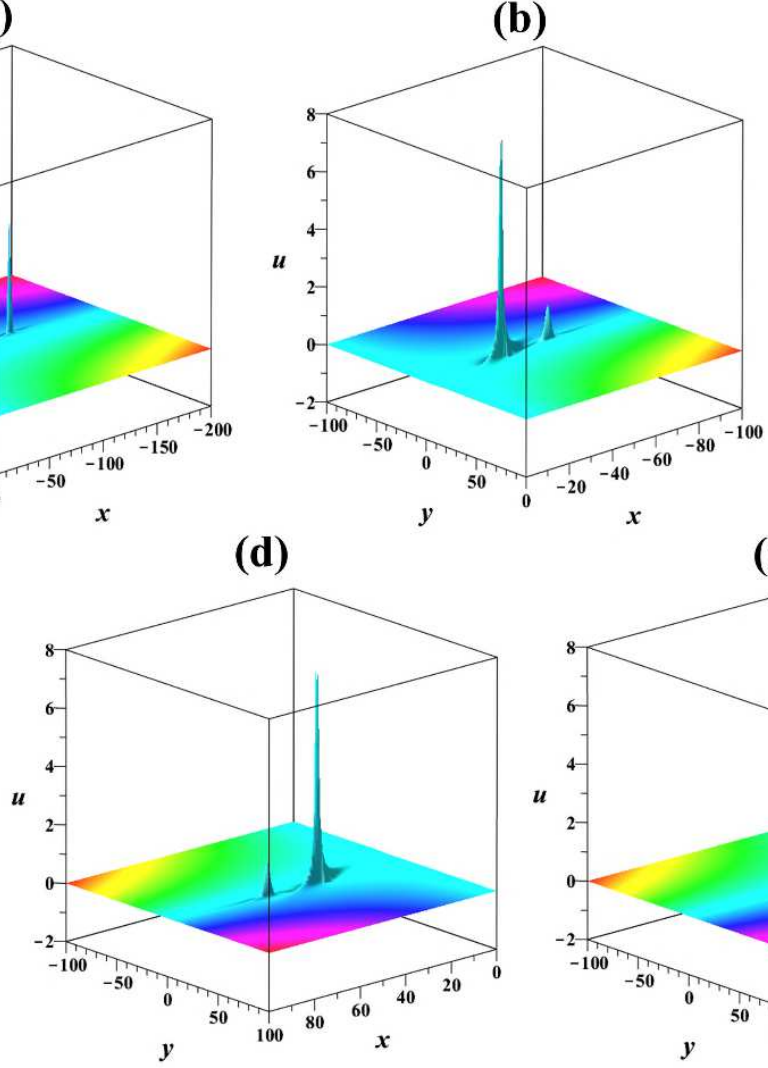

(e)

(c)
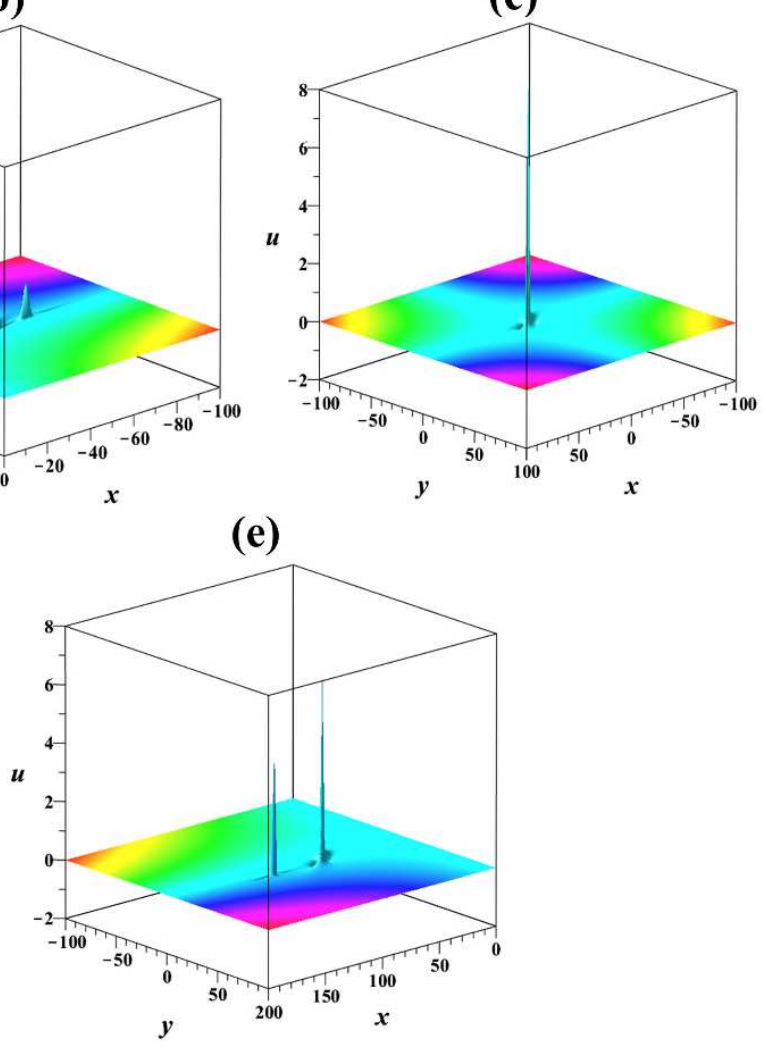

Figure 13. Time evolution 3D plots of the interaction solutions between two lump soliton solutions given by Eq. (51) at (a) $t=-50$, (b) $t=-25$, (c) $t=0$, (d) $t=25$, and (e) $t=50$ under constraint parameters as $m_{1}=m_{2}^{*}=-1+I, m_{3}=m_{4}^{*}=-1+10 I, \alpha=1, \beta=1$, $848 \gamma=1$, and $\delta=1$. 